\title{
WAVE MOTION IN A CONDUCTING FLUID WITH A LAYER ADJACENT TO THE BOUNDARY, II. EIGENFUNCTION EXPANSIONS
}

\author{
WILLIAM V. SMITH ${ }^{1}$
}

(Received 24 July 1997; revised 29 July 1998)

\begin{abstract}
The wave motion of magnetohydrodynamic (MHD) systems can be quite complicated. In order to study the motion of waves in a perfectly conducting fluid under the influence of an external magnetic field in a stratified medium, we make the simplifying assumption that the pressure is constant (to first order). This is the simplest form of the equations with variable coefficients and is not strongly propagative. Alfven waves are still present. The system is further simplified by assuming that the external field is parallel to the boundary. The Green's function for the operator is constructed and then the spectral family is constructed in terms of generalized eigenfunctions, giving four families of propagating waves, including waves "trapped" in the boundary layer. These trapped waves are interesting, since they are not the relics of surface waves, which do not exist in this context when the boundary layer shrinks to zero thickness no matter what (maximal energy preserving) boundary condition is chosen. We conjecture a similar structure for the full MHD problem.
\end{abstract}

\section{Introduction}

This paper continues our study [8] of linearized equations of magnetohydrodynamics in the setting of a medium filling a half-space with the presence of a slab or layer of "different" media near the boundary. This is what is meant by a "boundary layer" in our discussion. This system arises when the interest is in small deviations from an equilibrium state. The pressure is assumed to be constant to first order; this is the classical "cold plasma" condition. Many examples of systems behaving as cold plasmas exist including interstellar clouds and portions of planetary atmospheres. The type of "two fluid" or stratified media system treated here is a simplified version of such cases and is important in other applications such as fusion reactors [5, p. 247]. In our study, we consider a stratified or layered medium as the given fixed geometry

\footnotetext{
'Mathematics Department, Brigham Young University, Provo, Utah 84602, USA.

(C) Australian Mathematical Society 2001, Serial-fee code 0334-2700/01
} 
and derive consequences from this assumption. This may be thought of as a local treatment of a different geometry (spherical, etc.). See [4] to see how the kind of simple geometry we consider here can be related to much more general situations.

The main result of the paper may be seen as conditions for the existence of trapped waves in the layer (this happens when the phase speed of the layer is less than that of the adjoining media-see the text following (2.5) for the definition of phase speed) with their attendant structure, although no surface waves exist at the boundary. Theorem 5.14 gives the result that, for initial data which can propagate, the solution is delivered in orthogonal parts consisting of waves trapped in the layer, magnetosonic waves and Alfven waves. One interesting result of this analysis is that the explicit formulation allows us to see in advance how to or not to "launch" waves of a particular sort by choosing the "right" initial data (see remarks below Lemma 5.5). Such information can be useful in understanding or maintaining stability. Theorems 5.6 and 5.14 contain the/ technically accurate statements of these results.

The results given here do not stand in isolation. We believe that the techniques developed here are extendible in a rigorous way to systems of greater complexity. The next step would be to drop the cold plasma condition. A more elementary treatment is possible for a system of the type considered here, but this is much less helpful in determining what happens in the case where the pressure is not assumed constant. For a system related to our problem see [ 5, p. $256 \mathrm{ff}]$.

Our study depends explicitly on the spectral theory of operators in Hilbert space, but we have tried to keep some of the technical analysis in the background so that technical requirements do not obscure the treatment. Two appendices contain most of the proofs of theorems and other technical details. Formulae in Appendix I are identified with a prefix $A$ as (A1.1). We acknowledge here the helpful remarks of the referees.

Part I of this paper [8] established some necessary facts about the system such as a characterization of data which propagates and it introduced some methods useful for wave motion problems posed in a stratified medium. The results from part I which are referenced in this paper will be reviewed very briefly below. In order to make reference easier for the reader, equations, theorems and definitions, etc. will be numbered as a continuation of part $I$. Hence the next section below will be numbered as Section 4 and formulae having number smaller than (4.1) are from part I and have the numbering given there to make it simple for the reader to reference part I.

This work is based on [6] where a problem is treated involving one plane boundary. While it seems to be true that "mixed" problems in exterior domains are relatively well understood, less seems to be known in cases like the one studied here where the boundary is unbounded. Again, in the present work, the geometry consists of a plane boundary with a slab-like layer next to it with a half-space on the other side of the layer. 
We will now review as briefly as possible some items of notation and some formulae from part I which are used in this paper.

The hyperbolic part of the linearized equations of MHD with the assumption of constant pressure can be written as [6]

$$
\begin{aligned}
\mu \frac{\partial \mathbf{H}}{\partial t} & =\nabla \times\left(\mathbf{v} \times \mathbf{H}_{0}\right), \\
\rho \frac{\partial \mathbf{v}}{\partial t} & =(\nabla \times \mathbf{H}) \times \mathbf{H}_{0} .
\end{aligned}
$$

The vector quantities $\mathbf{v}, \mathbf{H}$ and $\mathbf{H}_{0}$ are the fluid velocity, magnetic field, and external (applied) magnetic field respectively. Normally the condition $\operatorname{div}(\mathbf{H})=0$ would be added, but in fact this condition is contained in $(0.1)$ in the sense that data which propagates essentially satisfies this condition. More details are found in [8] where we also consider various cases for the external field.

Here our results will focus on the case where $\mathbf{H}_{0}$ is constant and parallel to the boundary, specifically, $\mathbf{H}_{0}$ will be the vector $\left(h_{1}, h_{2}, h_{3}\right)$ with the choice $h_{1}=h_{3}=0$, $h_{2}=1$. In this case the choice of boundary conditions which are (maximal) energy conserving is more limited than in other cases [8].

In the above equations, $\nabla=\left(\partial / \partial x_{1}, \partial / \partial x_{2}, \partial / \partial x_{3}\right)$. The fluid is assumed to fill a half-space written as $\mathbf{R}_{-a}^{3}=\left\{x \in \mathbf{R}^{3}: x_{3}>-a\right\}, 0<a<\infty$, where the boundary $\left\{x_{3}=-a\right\}$ is energy preserving [8] while the density and magnetic permeability are given by $\rho, \rho_{0}$ and $\mu, \mu_{0}$ in the layer $\mathbf{R}^{2} \times(-a, 0)$ and the half space $\mathbf{R}_{+}^{3}=\left\{x \in \mathbf{R}^{3}: x_{3}>0\right\}$ respectively. To formulate this problem, let $E_{0}$ and $E$ denote the $6 \times 6$ matrices

$$
E_{0}=\operatorname{diag}\left(\rho_{0} I_{3 \times 3}, \mu_{0} I_{3 \times 3}\right) \quad \text { and } \quad E=\operatorname{diag}\left(\rho I_{3 \times 3}, \mu I_{3 \times 3}\right)
$$

and define $E(x)=\chi_{+}\left(x_{3}\right) E_{0}+\chi_{-}\left(x_{3}\right) E$, where $\chi_{+}$and $\chi_{-}$are the characteristic functions of $\mathbf{R}_{+}=\{x: x>0\}$ and $(-a, 0)$ respectively. Let $A(D), D_{j}=-i \partial / \partial x_{j}$, $j=1,2,3(i=\sqrt{-1})$, be the $6 \times 6$ matrix differential operator

$$
A(D)=\sum_{j} A_{j} D_{j}
$$

Our treatment differs from many formulations since we prefer to write $(0.1)$ in matrix form rather than in a Lagrangian form. We write $(0.1)$ in terms of the matrices $A_{j}$ as

$$
E \frac{\partial u}{\partial t}=\sum_{j=1}^{3} A_{j} \frac{\partial u}{\partial x_{j}}
$$


The matrices $A_{j}$ are given by

$$
\begin{aligned}
& A_{1}=\left[\begin{array}{cccccc}
0 & 0 & 0 & 0 & -h_{2} & -h_{3} \\
0 & 0 & 0 & 0 & h_{1} & 0 \\
0 & 0 & 0 & 0 & 0 & h_{1} \\
0 & 0 & 0 & 0 & 0 & 0 \\
-h_{2} & h_{1} & 0 & 0 & 0 & 0 \\
-h_{3} & 0 & h_{1} & 0 & 0 & 0
\end{array}\right] \text { and } \\
& A_{2}=\left[\begin{array}{cccccc}
0 & 0 & 0 & h_{2} & 0 & 0 \\
0 & 0 & 0 & -h_{1} & 0 & -h_{3} \\
0 & 0 & 0 & 0 & 0 & h_{2} \\
h_{2} & -h_{1} & 0 & 0 & 0 & 0 \\
0 & 0 & 0 & 0 & 0 & 0 \\
0 & -h_{3} & h_{2} & 0 & 0 & 0
\end{array}\right]
\end{aligned}
$$

As already mentioned, $h_{1}$ and $h_{3}$ are taken to be zero with $h_{2}=1$.

Formulae appearing below are numbered as they appear in Sections 2 and 3 of [8]. The linearized equations of constant pressure magnetohydrodynamics in the layered half-space may now be written as

$$
i \partial_{t} u(x, t)=E^{-1}(x) A(D) u(x, t) \equiv \Lambda^{\prime}(D) u(x, t) ;
$$

where $u(x, t)$ is the six-dimensional (column) vector $(\mathbf{v}, \mathbf{H}), \mathbf{v}$ is the fluid velocity and $\mathbf{H}$ is the magnetic field vector.

We will write $\Lambda^{\prime}(D)$ as

$$
\Lambda^{\prime}(D)=\chi_{+} \Lambda^{0}(D)+\chi_{-} \Lambda(D)=\chi_{+} E_{0}^{-1} A(D)+\chi_{-} E^{-1} A(D) .
$$

The problem now is to find a solution of (2.1) which is square-summable on $\mathbf{R}_{-a}^{3}$ for each $t$ and satisfies the initial and boundary conditions (see [8] for a detailed discussion of boundary conditions which conserve energy)

$$
\begin{gathered}
u(x, 0)=u_{0}(x), \\
\mathfrak{B} u\left(x_{1}, x_{2},-a, t\right)=0, \\
\mathfrak{B}=[0,0,0,0,1,0],
\end{gathered}
$$

for an external field $(0,1,0)$. It means that the $x_{2}$ component of the magnetic field must vanish at the boundary $x_{3}=-a$. Another energy preserving boundary operator in this case is given by $\mathfrak{B}_{1}=[0,0,1,0,0,0]$, which says that the vertical component of velocity is zero at the boundary. We refer the reader to Theorems 1.4 and 3.7 of [8].

Let $\mathscr{H}$ be the space $L_{2}\left(\mathbf{R}^{3}, \mathbf{C}^{6}\right)$, with the $E$ inner product: $(f, g)_{\mathscr{H}}=\langle f, E g\rangle$ $\left(\langle\cdot, \cdot\rangle\right.$ representing the usual $L_{2}$ inner product). $\mathscr{S}^{\prime}$ will denote the dual of the space 
$\mathscr{S}=\mathscr{S}\left(\mathbf{R}^{n}, \mathbf{C}^{6}\right)$ of rapidly decreasing smooth functions on $\mathbf{R}^{n}$, with values in $\mathbf{C}^{6}$. The Fourier transform

$$
\Phi_{n} f(p)=(2 \pi)^{-n / 2} \int_{\mathbf{R}^{n}} \exp (-i p \cdot x) f(x) d x
$$

is an automorphism of $\mathscr{S}$ with inverse $\Phi_{n}^{*} f(p)=\Phi_{n} f(-p)$ which extends by continuity to an automorphism of $L_{2}\left(\mathbf{R}^{3}, \mathbf{C}^{6}\right)$ and by duality to an automorphism of $\mathscr{S}^{\prime}$. For any $f$ in $\mathscr{H}$ the quantity $\Lambda(D) f \in \mathscr{S}^{\prime}$, and an operator with domain $\mathscr{D}(\Lambda)=\{f \in \mathscr{H}: \Lambda f \in \mathscr{H}\}$ is selfadjoint with resolvent $S(z)=(\Lambda-z I)^{-1}$ given by

$$
S(z) f(x)=\int_{\mathbf{R}^{3}} S(x, y ; z) f(y) d y .
$$

$\operatorname{Im}(z) \neq 0$ and $S(x, y ; z)=S(x-y ; z)$ is the fundamental solution

$$
[\Lambda(D)-z I] S(x ; z)=\delta(x) I
$$

where $l$ may be obtained from

$$
S(\cdot ; z)=(2 \pi)^{-3 / 2} \Phi^{*}[\Lambda(\cdot)-z I]^{-1} \Phi .
$$

Here we give some properties of $\Lambda^{0}$ and $\Lambda$. These will be stated for $\Lambda$. The corresponding properties for $\Lambda^{0}$ may be obtained by just affixing the index ${ }^{0}$ to all quantities containing the medium parameters. The transpose of a matrix $M$ is denoted by ${ }^{\prime} M$ and the conjugate transpose by ${ }^{\top} M$ while the adjoint of an operator is denoted by $M^{*}$. The matrices $E$ and $E_{0}$ generate equivalent inner products in $\mathbf{C}^{6}$ by the rule $E(x, y)=E x \circ y={ }^{\bar{t}} x E y$. In this inner product, the symbol $\Lambda(p)$ of $\Lambda(D)$ is given by $E^{-1} A(p)$ with

$$
A(p)=\left[\begin{array}{cccccc}
0 & 0 & 0 & p_{2} & -p_{1} & 0 \\
0 & 0 & 0 & 0 & 0 & 0 \\
0 & 0 & 0 & 0 & -p_{3} & p_{2} \\
p_{2} & 0 & 0 & 0 & 0 & 0 \\
-p_{1} & 0 & -p_{3} & 0 & 0 & 0 \\
0 & 0 & p_{2} & 0 & 0 & 0
\end{array}\right] .
$$

$A(p)$ is symmetric. $\Lambda(p)$ has the eigenvalues $\left(p=\left(p_{1}, p_{2}, p_{3}\right)\right.$ not zero in $\left.\mathbf{R}^{3}\right)$ $\lambda_{0}(p)=0$ with multiplicity two (the stationary speed),

$\lambda_{ \pm 1}(p)= \pm p_{2} /(\sqrt{\rho} \sqrt{\mu})$ (Alfven speeds), $\lambda_{ \pm 2}(p)= \pm|p| /(\sqrt{\rho} \sqrt{\mu})$ (magnetospeeds).

Let us define the phase speed $c$ by $(\sqrt{\rho} \sqrt{\mu})=c^{-1}$. 
The nonzero eigenvalues above each have multiplicity one for almost all $p$. Both the Alfven speed and the magnetospeed may coincide for some $p$, hence this system is not uniformly propagative. We see that it also fails to be strongly propagative (for definitions of uniformly propagative and strongly propagative see [9, p. 327] and $\left[10\right.$, p. 36]), since $\lambda_{ \pm 1}(p)$ may vanish for non-zero $p$. There exists a set of Lebesgue measure zero, $\beta$, such that when $p \in \mathbf{R}^{3} \backslash \beta$, the eigenvalues $\lambda_{0}(p), \lambda_{ \pm 1}(p), \lambda_{ \pm 2}(p)$ do not coincide [6]. We shall generally assume $p \in \mathbf{R}^{3} \backslash \beta$. Associated with each of the eigenvalues are mutually orthogonal eigenprojectors $P_{ \pm j}(p), P_{0}(p)$ with respect to the $E$ inner product. They generate the resolution of the identity for $\Lambda(p)$ :

$$
\begin{aligned}
I & =P_{0}(p)+P_{1}(p)+P_{-1}(p)+P_{2}(p)+P_{-2}(p), \\
\Lambda(p) & =\lambda_{1}(p) P_{1}(p)+\lambda_{-1}(p) P_{-1}(p)+\lambda_{2}(p) P_{2}(p)+\lambda_{-2}(p) P_{-2}(p) .
\end{aligned}
$$

The $P_{ \pm j}(p)$ satisfy the identities

$$
{ }^{\bar{\imath}}\left(E P_{ \pm j}\right)=E P_{ \pm j}, \quad \delta_{j k} P_{k}=P_{j} P_{k}, \quad \Lambda(p) P_{ \pm j}(p)=\lambda_{ \pm j}(p) P_{ \pm j}(p) .
$$

The functions $P$ will be needed explicitly. It is helpful to write " $z$ " in place of $\pm \lambda_{j}=\lambda_{ \pm j}$ as appropriate. Let the notation $|p|^{2}$ represent $p_{1}^{2}+p_{2}^{2}+p_{3}^{2}$ and $|p|_{1}^{2}$ stand for $p_{1}^{2}+p_{3}^{2}$. Then

$$
P_{ \pm 2 z}(p)=\frac{1}{2 \mu|p|_{1}^{2}|p|^{2}} \times
$$

$$
\begin{gathered}
{\left[\begin{array}{ccccccc}
\mu p_{1}^{2}|p|^{2} & 0 & \mu p_{1} p_{3}|p|^{2} & \mu^{2} p_{1}^{2} p_{2} z & -\mu^{2} p_{1}|p|_{1}^{2} z & \mu^{2} p_{1} p_{2} p_{3} z \\
0 & 0 & 0 & 0 & 0 & 0 \\
\mu p_{1} p_{3}|p|^{2} & 0 & \mu p_{3}^{2}|p|^{2} & \mu^{2} p_{1} p_{2} p_{3} z & -\mu^{2} p_{3}|p|_{1}^{2} z & \mu^{2} p_{2} p_{3}^{2} z \\
\mu p_{1}^{2} p_{2} \rho z & 0 & \mu p_{1} p_{2} p_{3} \rho z & \mu p_{1}^{2} p_{2}^{2} & -\mu p_{1} p_{2}|p|_{1}^{2} & \mu p_{1} p_{2}^{2} p_{3} \\
-\mu p_{1}|p|_{1}^{2} \rho z & 0 & -\mu p_{3}|p|_{1}^{2} \rho z & -\mu p_{1} p_{2}|p|_{1}^{2} & \mu|p|_{1}^{4} & -\mu p_{2} p_{3}|p|_{1}^{2} \\
\mu p_{1} p_{2} p_{3} \rho z & 0 & \mu p_{2} p_{3}^{2} \rho z & \mu p_{1} p_{2}^{2} p_{3} & -\mu p_{2} p_{3}|p|_{1}^{2} & \mu p_{2}^{2} p_{3}^{2}
\end{array}\right],} \\
P_{ \pm 1 z}(p)=\frac{1}{2 p_{2}|p|_{1}^{2}}\left[\begin{array}{ccccccc}
p_{2} p_{3}^{2} & 0 & -p_{1} p_{2} p_{3} & \mu p_{3}^{2} z & 0 & -\mu p_{1} p_{3} z \\
0 & 0 & 0 & 0 & 0 & 0 \\
-p_{1} p_{2} p_{3} & 0 & p_{1}^{2} p_{2} & -\mu p_{1} p_{3} z & 0 & \mu p_{1}^{2} z \\
p_{3}^{2} \rho z & 0 & -p_{1} p_{3} \rho z & p_{2} p_{3}^{2} & 0 & -p_{1} p_{2} p_{3} \\
0 & 0 & 0 & 0 & 0 & 0 \\
-p_{1} p_{3} \rho z & 0 & p_{1}^{2} \rho z & -p_{1} p_{2} p_{3} & 0 & p_{1}^{2} p_{2}
\end{array}\right], \quad(2.9) \\
P_{0}(p)=\frac{1}{|p|^{2}}\left[\begin{array}{ccccccc}
0 & 0 & 0 & 0 & 0 & 0 \\
0 & |p|^{2} & 0 & 0 & 0 & 0 \\
0 & 0 & 0 & 0 & 0 & 0 \\
0 & 0 & 0 & p_{1}^{2} & p_{1} p_{2} & p_{1} p_{3} \\
0 & 0 & 0 & p_{1} p_{2} & p_{2}^{2} & p_{2} p_{3} \\
0 & 0 & 0 & p_{1} p_{3} & p_{2} p_{3} & p_{3}^{2}
\end{array}\right] .
\end{gathered}
$$


$[\Lambda(\cdot)-z I]^{-1}$ may be determined by the spectral theorem from

$$
[\Lambda(p)-z I]^{-1}=\sum\left[\lambda_{ \pm k}(p)-z\right]^{-1} P_{ \pm k}(p) .
$$

To proceed, we need explicit formulae for $S(\cdot ; z)$ on hyperplanes orthogonal to the $x_{3}$ axis. To get these formulae, write $p=(\xi, \eta), \xi \in \mathbf{R}^{2}$, and extend $\lambda_{ \pm k}(p)$ to complex $\eta$ by the requirement $\pm \operatorname{Re} \lambda_{ \pm k}(p) \geq 0$ upon replacing $p$ by $(\xi, \tau)$ where $\tau=\eta+i \kappa$. Then $\lambda_{ \pm k}(p)$ becomes $\lambda_{ \pm k}(\xi, \tau)$ with $P_{ \pm k}(\xi, \tau)$ satisfying

$$
\Lambda(\xi, \tau) P_{ \pm k}(\xi, \tau)=\lambda_{ \pm k}(\xi, \tau) P_{ \pm k}(\xi, \tau) .
$$

For $\xi$ not zero define

$$
\tau_{ \pm 2}(\xi, z)=c^{-1}\left(z^{2}-c^{2}|\xi|^{2}\right)^{1 / 2},
$$

where $\pm \operatorname{Im} \tau_{ \pm k} \geq 0$ in the $z$-plane with branch cuts $(-\infty,-c|\xi|),(c|\xi|, \infty)$. Note that $\lambda_{ \pm 1}(\xi, \tau)$ is a constant function of $\tau$. Observe that

$$
\tau_{+2}(\xi, z)=-\tau_{-2}(\xi, z), \quad \bar{\tau}_{ \pm 2}(\xi, \bar{z})=-\tau_{ \pm 2}(\xi, z) .
$$

The matrix $[\Lambda(\xi, \tau)-z I]^{-1}$ is regular in $\tau$ except for poles in the upper (lower) half plane at the zeroes of $\operatorname{det}[\Lambda(\xi, \tau)-z I]$, that is at $\tau_{+k}\left(\tau_{-k}\right)$, and in a neighborhood of these poles

$$
[\Lambda(\xi, \tau)-z I]^{-1}=\sum\left[\lambda_{ \pm k}(\xi, \tau)-z\right]^{-1} P_{ \pm k}(\xi, \tau) .
$$

If we now apply $\Phi_{2}$ to both sides of (2.12), we obtain, in $\mathscr{S}^{\prime}$, the relation

$$
\Phi_{2} S\left(\xi, x_{3} ; y ; z\right)=(2 \pi)^{-2} e^{-i y^{\prime} \xi} \int_{\mathbf{R}} e^{i\left(x_{3}-y_{3}\right)}[\Lambda(\xi, \tau)-z I]^{-1} d \tau .
$$

In order to simplify the notation in the evaluation of this integral, we shall from here on employ the definitions $\tau \equiv \tau_{+}, \tau^{0} \equiv \tau_{+}^{0}$. An elementary computation gives

$$
\begin{aligned}
\Phi_{2} S(\xi,-a ; y ; z) & =i(2 \pi)^{-1} c^{-2} e^{-i y^{\prime} \xi} e^{i \tau\left(a+y_{3}\right)} z \tau^{-1} P(\xi, z,-\tau), \\
\Phi_{2} S(\xi, 0 ; y ; z) & =i(2 \pi)^{-1} c^{-2} e^{-i y^{\prime} \xi} e^{i \tau y_{3}} z \tau^{-1} P(\xi, z,-\tau), \quad-a<y_{3}<0, \\
\Phi_{2} S^{0}(\xi, 0 ; y ; z) & =i(2 \pi)^{-1} c_{0}^{-2} e^{-i y^{\prime} \xi} e^{i \tau^{0} y_{3}} z \tau^{0^{-1}} P^{0}\left(\xi, z,-\tau^{0}\right), \quad 0<y_{3} .
\end{aligned}
$$

Here $P(\xi, z, \tau)$ is given explicitly by $(2.8)$ (with $p_{3}$ replaced by $\tau$ ). In actual fact, a trivial delta function term must be added to these functions to acount for the projections $P_{ \pm 1}$. See (5.29)-(5.31) below for this term.

$P(\xi, z, \tau)$ is a solution of

$$
\Lambda(\xi, \tau) P(\xi, z, \tau)=z P(\xi, z, \tau) .
$$


$P^{0}\left(\xi, z, \tau^{0}\right)$ is obtained from (2.8) by replacing $\rho$ and $c$ with $\rho_{0}$ and $c_{0}$ respectively. It satisfies

$$
\Lambda^{0}(\xi, \tau) P^{0}\left(\xi, z, \tau^{0}\right)=z P^{0}\left(\xi, z, \tau^{0}\right)
$$

The identities

$$
\begin{aligned}
(E P(\xi, \bar{z}, \tau(\xi, \bar{z}))) & =E P(\xi, z,-\tau(\xi, z)) \quad \text { and } \\
\left(E_{0} P^{0}\left(\xi, \bar{z}, \tau^{0}(\xi, \bar{z})\right)\right) & =E_{0} P^{0}\left(\xi, z,-\tau^{0}(\xi, z)\right)
\end{aligned}
$$

hold.

The following results, proved in part I, are needed here.

THEOREM 3.7. $\Lambda^{\prime}$ is a selfadjoint operator in $\mathscr{K}\left(\mathscr{K}\right.$ is the space $L_{2}\left(\mathbf{R}_{-\mathfrak{a}}^{3}, \mathbf{C}^{6}\right)$ with the $E$ inner product).

The interface condition used in this paper is condition (3.6) from part I. It is given by $\left(L f={ }^{\prime}\left(f_{3}, f_{5}\right)\right)$

$$
L f(\circ, 0-)=L f(\circ, 0+) \text {, }
$$

in the sense of $\mathbf{H}^{-1 / 2}$. Note that this can be seen as a consequence of assuming that $\Lambda^{\prime}$ is selfadjoint:

$$
0=\left(\Lambda^{\prime} f, \phi\right)_{\mathscr{X}}-\left(f, \Lambda^{\prime} \phi\right)_{\mathscr{K}}=i\left[\left\{A_{3} f(\circ, 0+)-A_{3} f(\circ, 0-)\right\}, \phi(\circ, 0)\right],
$$

for $f \in D\left(\Lambda^{\prime}\right)$ (the domain of $\left.\Lambda^{\prime}\right), \phi \in \mathscr{D}\left(\mathbf{R}_{-a}^{3}, \mathbf{C}^{6}\right)$, and thus $A_{3} f(\circ, 0+)=$ $A_{3} f(\circ, 0-)$ in the sense of Sobolev space $\mathbf{H}^{-1 / 2}$. If $f$ also is smooth (say, $f \in D\left(A^{0}\right)$ ) then (3.6) holds pointwise.

PROPOSITION 3.10. A function $f={ }^{t}\left(f_{1}, f_{2}, f_{3}, f_{4}, f_{5}, f_{6}, f_{7}\right)$ in $\mathscr{K}$ is orthogonal in $\mathscr{K}$ to $N\left(\Lambda^{\prime}\right)$ (the null space of $\left.\Lambda^{\prime}\right)$ if and only if

$$
\begin{array}{ll}
f_{2}=0 \quad \text { and } \quad \operatorname{div}\left(f_{4}, f_{5}, f_{6}\right)=0 \quad \text { in } L_{2}\left(\mathbf{R}_{+}^{3}, \mathbf{C}^{6}\right) \text { and } \\
& \text { in } L_{2}\left(\mathbf{R}^{2} \times(-a, 0), \mathbf{C}^{6}\right)
\end{array}
$$

and

$$
\begin{gathered}
(E f)_{6}\left(x^{\prime}, 0-\right)=(E f)_{6}\left(x^{\prime}, 0+\right), \\
(E f)_{6}(0,-a)=0,
\end{gathered}
$$

in $\mathrm{H}^{-1 / 2}$.

This gives us sufficient background to study the resolvent operator for $\Lambda^{\prime}$. 


\section{The resolvent of $\Lambda^{\prime}$}

The purpose of this section is to work out the detailed analytic structure of a certain Green's matrix. To be more specific, the problem of representing the solution of (2.1), that is, (2.3), in terms of the various modes of propagation reduces, by the spectral theorem, to representing the spectral family of $\Lambda^{\prime}$ in terms of these modes. This in turn reduces to finding a convenient form for the resolvent of $\Lambda^{\prime}$. The method was used first in a similar setting by Ikebe [3]. Explicitly, if $u(x, t)$ is the solution of (2.1), that is, (2.3) with initial value $u_{0}(x)$, then the solution $u$ is delivered by a unitary group $U(t)$ in $\mathscr{K}, u(x, t)=U(t) u_{0}(x)$ (see Theorem 5.14 below). If $F_{\lambda}=F(-\infty, \lambda)=F(\lambda)$ is the resolution of the identity for $\Lambda^{\prime}$, then $(F(\lambda)$ is taken to be continuous)

$$
U(t)=\int_{\mathbf{R}} \exp (-i t \lambda) d F_{\lambda} .
$$

$F_{\lambda}$ is obtained from $G(z)=\left(\Lambda^{\prime}-z I\right)^{-1}$ by Stone's formula:

$$
\frac{F(b)+F(b-)}{2}-\frac{F(a)+F(a-)}{2}=\frac{1}{2 \pi i} \lim _{\varepsilon \downarrow 0} \int_{a}^{b}[G(\lambda+i \varepsilon)-G(\lambda-i \varepsilon)] d \lambda .
$$

See Theorem 5.6 for the complete structure in (4.2).

To carry out this program, it is necessary to express the limit of $[G(\lambda+i \varepsilon)-$ $G(\lambda-i \varepsilon)]$ in terms of the modes of propagation in order to so express $u(x, t)$. As advertised, this is done by considering an appropriate representation of the resolvent kernel, $G(x, y ; z)$ :

$$
G(z) f(x)=\int_{\mathbf{R}_{-a}^{3}} G(x, y ; z) f(y) d y .
$$

This suggests that the columns of $G(x, y ; z)$ should satisfy the boundary condition of (2.3) and also the interface condition (3.6) at $x_{3}=0$ (that is, we expect $G(z) f$ to belong to $D\left(\Lambda^{\prime}\right)$ ). Further, since $\Lambda^{\prime}$ is selfadjoint in $\mathscr{K}$, the identity $G(z)^{*}=G(\bar{z})$ must hold. Hence $(G(z) f, g)=\left(f, G(z)^{*} g\right)=(f, G(\bar{z}) g)$ and therefore

$$
\int\left[\int(G(x, y ; z) f(y) d y] E(x) g(x) d x=\int{ }^{\bar{t}} f(y) \int E(y) G(x, y ; \bar{z}) g(x) d x d y .\right.
$$

It follows that

$$
E(x) G(x, y ; z)={ }^{\bar{\imath}} G(x, y ; \bar{z}) E(y) .
$$

The interpretation of $G(x, y ; z)$ as the stationary field at point $x$ resulting from a superposition of unit point sources with coefficients $f(y), y$ in the support of $f(y)$, further suggests that $G(x, y ; z)$ be written in a form

$$
G(x, y ; z)=\chi_{+}\left(y_{3}\right) G(x, y ; z)+\chi_{-}\left(y_{3}\right) G(x, y ; z)
$$




$$
\equiv G_{+}(x, y ; z)+G_{-}(x, y ; z) \text {. }
$$

$G_{+}(x, y ; z)$ and $G_{-}(x, y ; z)$ correspond to this field, respectively above and below the plane $x_{3}=0$. They each contain a term associated with the $P_{ \pm 1}$ projectors of the form consisting of kernels generated by $\chi_{-} \Phi^{*}\left(\lambda_{ \pm}(p)-z\right)^{-1} P_{ \pm 1}(p) \Phi \chi_{-}, \chi_{+} \Phi^{*}\left(\lambda_{ \pm}^{0}(p)-\right.$ $z)^{-1} P_{ \pm 1}^{0}(p) \Phi_{\chi_{+}}$. These Alfven wave terms are just delta function terms and the part of the solution they generate is elementary (see [6]) and is given later (see (5.29)-(5.31) below). The equation $[\Lambda(D)-z I] S(x ; z)=\delta(x) I$, and elementary physics, suggest seeking $G_{+}(x, y ; z)$ in the special form

$$
\begin{aligned}
G_{+}(x, y ; z) & =\chi_{+}\left(y_{3}\right) G(x, y ; z) \\
& =\chi_{+}\left(y_{3}\right)\left\{\chi_{+}\left(x_{3}\right)\left[S^{0}(x, y ; z)-R^{0}(x, y ; z)\right]+\chi_{-}\left(x_{3}\right) T(x, y ; z)\right\}
\end{aligned}
$$

where $S^{0}(x, y ; z)=S^{0}(x,-y ; z)$ is the fundamental solution for $\Lambda^{0}(D)$ which may be pictured as incident radiation from the point source at $y$, while $R^{0}(x, y ; z)$ represents the field reflected from the interface and $T(x, y ; z)$ the field transmitted through the interface $x_{3}=0$. They should satisfy the equations

$$
\begin{aligned}
& {\left[\Lambda^{0}\left(D_{x}\right)-z I\right] R^{0}(x, y ; z)=0, \quad x_{3}, y_{3} \in \mathbf{R}_{+} \text {, }} \\
& {\left[\Lambda\left(D_{x}\right)-z I\right] T(x, y ; z)=0, \quad y_{3} \in \mathbf{R}_{+}, x_{3} \in(-a, 0) \text {, }}
\end{aligned}
$$

the boundary condition

$$
\mathfrak{B} T\left(x^{\prime},-a, y ; z\right)=0
$$

and the interface condition

$$
L S^{0}\left(x^{\prime}, 0+, y ; z\right)=L R^{0}\left(x^{\prime}, 0+, y ; z\right)+L T\left(x^{\prime}, 0-, y ; z\right)
$$

In (4.8) and henceforth, $f\left(x^{\prime},-a\right)$ means $f\left(x^{\prime},-a+0\right)$. Taking the Fourier transform on the tangential variables $x^{\prime}$, conditions (4.7)-(4.9) become

$$
\begin{gathered}
{\left[\Lambda^{0}\left(\xi, D_{x_{3}}\right)-z I\right] \Phi_{2} R^{0}\left(\xi, x_{3}, y ; z\right)=0, \quad x_{3}, y_{3} \in \mathbf{R}_{+},} \\
{\left[\Lambda\left(\xi, D_{x_{3}}\right)-z I\right] \Phi_{2} T\left(\xi, x_{3}, y ; z\right)=0, \quad y_{3} \in \mathbf{R}_{+}, x_{3} \in(-a, 0)} \\
\mathfrak{B} \Phi_{2} T(\xi,-a, y ; z)=0 \\
L \Phi_{2} S^{0}(\xi, 0+, y ; z)=L \Phi_{2} R^{0}(\xi, 0+, y ; z)+L \Phi_{2} T(\xi, 0-, y ; z)
\end{gathered}
$$

Equations (4.10)-(4.12) are satisfied by

$$
\begin{aligned}
& \Phi_{2} R^{0}\left(\xi, x_{3}, y ; z\right)=\alpha(\xi, y, z) e^{i \tau^{0} x_{3}} P^{0}\left(\xi, z, \tau^{0}\right) C^{0}(\xi, z) \\
& \Phi_{2} T\left(\xi, x_{3}, y ; z\right)=\alpha(\xi, y, z) c_{0}^{2} c^{-2}\left\{e^{-i z\left(x_{3}+a\right)} P(\xi, z,-\tau)\right.
\end{aligned}
$$




$$
\begin{gathered}
\left.-e^{i \tau\left(x_{3}+a\right)} P(\xi, z, \tau) Q_{a}\right\} D^{\prime}(\xi ; z), \\
\Phi_{2} T\left(\xi, x_{3}, y ; z\right)=\alpha(\xi, y, z) c_{0}^{2} c^{-2} Q\left(\tau\left(x_{3}+a\right)\right) P(\xi, z,-\tau) D^{\prime}(\xi ; z) .
\end{gathered}
$$

$Q_{a}$ is the matrix $\operatorname{diag}(1, A,-1,1,1,-1)$ and $\alpha(\xi, y, z)$ is a function chosen to simplify the computations needed later, $\alpha(\xi, y, z)=i(2 \pi)^{-1} c_{0}^{-2} e^{-i y^{\prime} \xi+i \tau^{0} y_{3}} z \tau^{0^{-1}}$. $Q(\phi)$ is the matrix $\operatorname{diag}(-s(\phi), A, c(\phi),-s(\phi),-s(\phi), c(\phi))$, with $s(\phi)=2 i \sin (\phi)$, $c(\phi)=2 \cos (\phi)$. The matrices $C^{0}(\xi, z)$ and $D^{\prime}(\xi ; z)$ will now be chosen to satisfy (4.13). The matrix $Q_{a}$ has the property ( $A$ is arbitrary)

$$
Q_{a} P(\xi, z,-\tau)=P(\xi, z, \tau) Q_{a}
$$

This ensures that condition (4.12) is satisfied. It remains to choose the matrices $C^{0}$ and $D^{\prime}$ to satisfy condition (4.13). (4.13) now becomes

$$
L P^{0}\left(\xi, z, \tau^{0}\right)=L P^{0}\left(\xi, z, \tau^{0}\right) C^{0}(\xi, z)+c_{0}^{2} c^{-2} L Q(a \tau) P(\xi, z, \tau) D^{\prime}(\xi ; z)
$$

There are diagonal matrix solutions to this equation of the form

$$
C^{0}(\xi, z)=\operatorname{diag}\left(k_{1}, A, k_{3}, k_{4}, k_{5}, k_{6}\right) \text { and } D^{\prime}(\xi ; z)=\operatorname{diag}\left(d_{1}, A, d_{3}, d_{4}, d_{5}, d_{6}\right) \text {. }
$$

Each of the entries $k_{1}, \ldots, k_{6}, d_{1}, \ldots, d_{6}$ are functions of $\xi$ and $z$; their full form is given in Appendix II.

At this point, we are mainly interested in the singularities of these matrix functions since these singularities will determine the important trapped wave modes. The denominators of the matrix entries are essentially the following functions as determined by elementary linear algebra:

$$
\begin{aligned}
K(\xi, z)= & \mu \tau \cos (a \tau)\left(p_{1}^{2}+\tau^{0^{2}}\right)-i \mu_{0} \tau^{0} \sin (a \tau)\left(p_{1}^{2}+\tau^{2}\right) \\
J(\xi, z)= & \tau \cos (a \tau) \rho_{0}\left(p_{1}^{4}+p_{1}^{2} p_{2}^{2}+\tau^{2} p_{1}^{2}+\tau^{0^{2}} p_{1}^{2}+\tau^{0^{2}} p_{2}^{2}+\tau^{2} \tau^{0^{2}}\right) \\
& -i \rho \sin (a \tau) \tau^{0}\left(p_{1}^{4}+p_{1}^{2} \tau^{2}+p_{1}^{2} p_{2}^{2}+p_{2}^{2} \tau^{2}+\tau^{0^{2}} p_{1}^{2}+\tau^{0^{2}} \tau^{2}\right) .
\end{aligned}
$$

( $J$ and $K$ should not be confused with the usual Bessel functions.) The zeroes of these two functions determine the singularities of the matrices. We therefore need some information about them.

By (2.15),

$$
\bar{K}(\xi, \bar{z})=-K(\xi, z), \quad \bar{J}(\xi, \bar{z})=-J(\xi, z)
$$

In fact, by (2.15),

$$
\tau_{+2}(\xi, z)=-\tau_{-2}(\xi, z), \quad \bar{\tau}_{ \pm 2}(\xi, \bar{z})=-\tau_{ \pm 2}(\xi, z)
$$


and so

$$
{ }^{\bar{i}} C^{0}(\xi, \bar{z})=C^{0}(\xi, z), \quad{ }^{\bar{t}} D^{\prime}(\xi, \bar{z})=D^{\prime}(\xi, z),
$$

as can be seen from their formulae given in Appendix II.

In a similar manner,

$$
\begin{aligned}
G_{-}(x, y ; z) & =\chi_{-}\left(y_{3}\right) G(x, y ; z) \\
& =\chi_{-}\left(y_{3}\right)\left\{\chi_{-}\left(x_{3}\right)[H(x, y ; z)-R(x, y ; z)]+\chi_{+}\left(x_{3}\right) T^{0}(x, y ; z)\right\},
\end{aligned}
$$

where $H=I-F$ is the resolvent kernel for the half-space $\mathbf{R}_{-a}^{3}$ which satisfies (2.13) and the boundary condition $(3.1), R(x, y ; z)$ is the reflected field in the layer and $T^{0}(x, y ; z)$ is the field transmitted to $x_{3}>0$. They satisfy

$$
\begin{aligned}
{\left[\Lambda\left(D_{x}\right)-z I\right] R(x, y ; z) } & =0, & & x_{3}, y_{3} \in(-a, 0), \\
{\left[\Lambda^{0}\left(D_{x}\right)-z I\right] T^{0}(x, y ; z) } & =0, & & y_{3} \in(-a, 0), x_{3} \in \mathbf{R}_{+},
\end{aligned}
$$

the boundary condition

$$
\mathfrak{B} H\left(x^{\prime},-a, y ; z\right)=\mathfrak{B R}\left(x^{\prime},-a, y ; z\right)=0
$$

and the interface condition

$$
L H\left(x^{\prime}, 0, y ; z\right)=L R\left(x^{\prime}, 0-, y ; z\right)+L T^{0}\left(x^{\prime}, 0+, y ; z\right) .
$$

Taking the Fourier transform on $x^{\prime}$,

$$
\begin{gathered}
\Phi_{2} H\left(\xi, x_{3}, y ; z\right)=\Phi_{2} S\left(\xi, x_{3}, y ; z\right)-\Phi_{2} F\left(\xi, x_{3}, y ; z\right), \\
{\left[\Lambda\left(\xi, D_{x_{3}}\right)-z I\right] \Phi_{2} R\left(\xi, x_{3}, y ; z\right)=0,} \\
{\left[\Lambda^{0}\left(\xi, D_{x_{3}}\right)-z I\right] \Phi_{2} T^{0}\left(\xi, x_{3}, y ; z\right)=0,} \\
\mathfrak{B} \Phi_{2} H(\xi,-a, y ; z)=\mathfrak{B} \Phi_{2} R(\xi,-a, y ; z)=0, \\
L \Phi_{2} H(\xi, 0, y ; z)=L \Phi_{2} R(\xi, 0-, y ; z)+L \Phi_{2} T^{0}(\xi, 0+, y ; z) .
\end{gathered}
$$

Employing $Q_{a}$ and $Q$ we have from (2.18), (2.19) and (2.20),

$$
\begin{aligned}
\Phi_{2} F\left(\xi, x_{3}, y ; z\right) & =\beta e^{i \tau y_{3}} e^{i t\left(x_{3}+a\right)} P(\xi, z, \tau) Q_{a}, \\
\Phi_{2} H(\xi,-a, y ; z) & =\beta e^{i t y_{3}}\left[I-Q_{a}\right] P(\xi, z, \tau), \\
\Phi_{2} H(\xi, 0, y ; z) & =\beta P(\xi, z, \tau) Q\left(\tau\left(y_{3}+a\right)\right), \\
\Phi_{2} R\left(\xi, x_{3}, y ; z\right) & =\beta\left[e^{-i\left(x_{3}+a\right) \tau} P(\xi, z,-\tau)-e^{i\left(x_{3}+a\right) \tau} P(\xi, z, \tau) Q_{a}\right] C(\xi, y ; z) \\
& =\beta Q\left(\tau\left(y_{3}+a\right)\right) P(\xi, z,-\tau) C(\xi, y ; z),
\end{aligned}
$$




$$
\begin{gathered}
\Phi_{2} T^{0}\left(\xi, x_{3}, y ; z\right)=\beta c^{2} c_{0}^{-2} e^{i \tau^{0} x_{3}} P^{0}\left(\xi, z, \tau^{0}\right) D^{0^{\prime \prime}}(\xi, y ; z), \\
\beta=\beta(x, y ; z)=i c^{-2}(2 \pi)^{-1} \exp \left(-i y^{\prime} x+i a \tau\right) z \tau^{-1} .
\end{gathered}
$$

( $\beta$ is obviously not the set $\beta$ defined after (2.5) and appearing in Theorem 4.1 below.) Both $\Phi_{2} H$ and $\Phi_{2} R$ satisfy the boundary condition. The matrices $C$ and $D^{\alpha^{\prime \prime}}$ are chosen to satisfy (4.28) which becomes

$$
L P(\xi, z, \tau) Q\left(\tau\left(y_{3}+a\right)\right)=L Q(a \tau) P(\xi, z,-\tau) C+c^{2} c_{0}^{-2} L P^{0}\left(\xi, z, \tau^{0}\right) D^{0^{\prime \prime}} .
$$

Once again, there are diagonal matrices $C$ and $D^{0^{\prime \prime}}$ which satisfy this equation and singularities are once again determined by the functions $J$ and $K$ above. The complete forms are given in Appendix II. We have

$$
{ }^{\overline{ }} C(\xi, y, \bar{z})=C(\xi, y, z), \quad{ }^{i} D^{0^{\prime \prime}}(\xi, y, \bar{z})=D^{0^{\prime \prime}}(\xi, y, z) .
$$

As noted in Appendix II,

$$
C(\xi, y, z)=Q\left(\tau\left(y_{3}+a\right)\right) C^{\prime}(\xi, z)=C^{\prime}(\xi, z) Q\left(\tau\left(y_{3}+a\right)\right)
$$

for the matrix $C^{\prime}(\xi, z)$ (with singularities determined by $J$ and $K$ ) given there. Also there is a matrix $D^{0^{\prime}}(\xi, z)$ (given in Appendix II) satisfying the equations

$$
\begin{aligned}
\tau^{0} \tau^{-1} e^{i a \tau} E^{-1} D^{0^{\prime \prime}}(\xi, y, z) E_{0} & =Q\left(\tau\left(y_{3}+a\right)\right) D^{0^{\prime}}(\xi, z) \\
& =D^{0^{\prime}}(\xi, z) Q\left(\tau\left(y_{3}+a\right)\right) .
\end{aligned}
$$

The two matrices $C^{\prime}$ and $D^{0^{\prime}}$ satisfy the identities (4.35) (see Appendix II). We are now able to obtain the facts (the terms corresponding to the projectors $P_{ \pm 1}$ are left out and will be given later, but see (5.29)-(5.31))

$$
\begin{aligned}
& E(x) G_{+}(x, y ; z)=\chi_{+}\left(y_{3}\right)^{\bar{t}}\left\{\chi_{+}\left(x_{3}\right)\left[I^{0}-R^{0}\right](y, x ; \bar{z})+\chi_{-}\left(x_{3}\right) T^{0}(y, x ; \bar{z})\right\} E_{0}, \\
& E(x) G_{-}(x, y ; z)=\chi_{-}\left(y_{3}\right)^{\bar{t}}\left\{\chi_{-}\left(x_{3}\right)[H-R](y, x ; \bar{z})+\chi_{+}\left(x_{3}\right) T(y, x ; \bar{z})\right\} E,
\end{aligned}
$$

or equivalently,

$$
\begin{aligned}
E(x) \Phi_{2} G_{+}\left(\xi, x_{3}, y ; z\right)= & e^{-i\left(x^{\prime}+y^{\prime}\right) \xi} \chi_{+}\left(y_{3}\right)^{\bar{t}}\left\{\chi_{+}\left(x_{3}\right) \Phi_{2}\left[I^{0}-R^{0}\right]\left(\xi, y_{3}, x ; \bar{z}\right)\right. \\
& \left.+\chi_{-}\left(x_{3}\right) \Phi_{2} T^{0}\left(\xi, y_{3}, x ; \bar{z}\right)\right\} E_{0} \\
E(x) \Phi_{2} G_{-}(\xi, x, y ; z)= & e^{-i\left(x^{\prime}+y^{\prime}\right) \xi} \chi_{-}\left(y_{3}\right)^{\bar{i}}\left\{\chi_{-}\left(x_{3}\right) \Phi_{2}[H-R]\left(\xi, y_{3}, x ; \bar{z}\right)\right. \\
& \left.+\chi_{+}\left(x_{3}\right) \Phi_{2} T\left(\xi, y_{3}, x ; \bar{z}\right)\right\} E .
\end{aligned}
$$

By (2.17), (4.15) and (4.18),

$$
e^{-i\left(x^{\prime}+y^{\prime}\right) \xi}\left[E_{0} \Phi_{2} S^{0}\left(\xi, y_{3}, x ; \bar{z}\right)\right]=E_{0} \Phi_{2} S^{0}\left(\xi, y_{3}, x ; z\right),
$$




$$
e^{-i\left(x^{\prime}+y^{\prime}\right) \xi}\left[E_{0} \Phi_{2} H\left(\xi, y_{3}, x ; \bar{z}\right)\right]=E_{0} \Phi_{2} H\left(\xi, y_{3}, x ; z\right)
$$

From (2.17), (4.15), (4.19), (4.36), (4.37) and Appendix II, we have the following important relations (called in [2] "paramutation relations"):

$$
\begin{aligned}
Q_{a} P(\xi, z, \tau) & =P(\xi, z,-\tau) Q_{a}, \\
Q_{a} P^{0}\left(\xi, z, \tau^{0}\right) & =P^{0}(\xi, z,-\tau) Q_{a}, \\
C^{0}(\xi, z) P^{0}\left(\xi, z,-\tau^{0}\right) & =P^{0}\left(\xi, z, \tau^{0}\right) C^{0}(\xi, z), \\
c_{0}^{-2} D^{0^{\prime}}(\xi, z) P^{0}\left(\xi, z,-\tau^{0}\right) & =c^{-2} P(\xi, z,-\tau) D^{\prime}(\xi, z), \\
C^{\prime}(\xi, z) P(\xi, z, \tau) & =P(\xi, z,-\tau) C^{\prime}(\xi, z) .
\end{aligned}
$$

The zeroes of the functions $J$ and $K$ give the frequencies of the "trapped waves". For each such zero there corresponds a generalized eigenfunction of $\Lambda^{\prime}$. Some properties of these functions are given in the following theorem.

THEOREM 4.1. (Recall that $p \notin \beta$.) The zeroes of the functions $J$ and $K$ are all real. In the case $c_{0} c^{-1}<-1$, these functions have no zeroes for $z \neq 0,|\xi| \neq 0$. In the case $n=c_{0} c^{-1}>1$, the zeroes of $J$ and $K$ respectively form two sequences $\left\{v_{j}(\xi)\right\}$ and $\left\{w_{j}(\xi)\right\}$. They have the following properties:

(1) $\left\{v_{j}(\xi)\right\}$ and $\left\{w_{j}(\xi)\right\}$ are defined for $|\xi|>r_{j}=(j+1 / 2) r_{0}, r_{0}=\pi a^{-1}\left(n^{2}-1\right)^{-1 / 2}$; and they are positive, bounded away from zero, and there are exactly $j+1$ roots for $r_{0}(j+1 / 2)<|\xi|<(j+3 / 2) r_{0}$;

(2) for each fixed $\xi$, the sequences $\left\{v_{j}(\xi)\right\},\left\{w_{j}(\xi)\right\}$ are finite and strictly increasing for each fixed $\xi_{1}, \xi_{2} ;$ they are contained in the interval $\left(c|\xi|, c_{0}|\xi|\right)$;

(3) $v_{j}, w_{j} \rightarrow c_{0} r_{j}$ and $v_{j}, w_{j}>c_{0} r_{j}$ for $|\xi|>r_{j}$. $\partial v_{j}$ and $\partial w_{j} \rightarrow c_{0}$ as $|\xi| \rightarrow r_{j}$. Thus $v_{j}$ and $w_{j}$ may be extended as $\mathbf{C}^{1}$ functions of $\xi$ to the left of $r_{j}$ by $c_{0}|\xi|$.

(For the proof, see Appendix I.)

The next theorem tells us that we are on the right track. The proof is given in Appendix I.

THEOREM 4.2. For $\operatorname{Im}(z) \neq 0$ the function $G(x, y ; z)$ gives a representation of the resolvent $G(z)$ for $\Lambda^{\prime}$, that is, if $g \in \mathscr{K}$ and

$$
f(x)=\int_{\mathbf{R}_{-a}^{3}} G(x, y ; z) g(y) d y,
$$

then $f \in D\left(\Lambda^{\prime}\right)$ and $\left[\Lambda^{\prime}-z I\right] f=g$.

Some important relations are given by the following two results. The proof of the first is found in Appendix I. 
LEMMA 4.3. For $G_{ \pm}(x, y ; z)$ above, and fixed $x \in \mathbf{R}_{-a}^{3}$ in the sense of $\mathscr{S}^{\prime}\left(\mathbf{R}_{y}^{3}\right)$,

$$
\begin{aligned}
& \left\{\Lambda\left(D_{y}\right)-\bar{z} I\right\} G_{+}(x, y ; z) \\
& =\chi_{+}\left(x_{3}\right)\left\{\delta(y-x)-i \delta\left(y_{3}\right) \Lambda_{3}^{0}\left[I^{0}\left(y^{\prime}, 0, x ; \bar{z}\right)-R^{0}\left(y^{\prime}, 0+, x ; \bar{z}\right)\right]\right\} \\
& \quad-i \chi_{-}\left(x_{3}\right) \Lambda_{3}^{0} \delta\left(y_{3}\right) T^{0}\left(y^{\prime}, 0+, x ; \bar{z}\right), \\
& \left\{\Lambda\left(D_{y}\right)-\bar{z} I\right\} G_{-}(x, y ; z) \\
& =\chi_{-}\left(x_{3}\right)\left(\delta(y-x)+i \Lambda_{3}\left\{\delta\left(y_{3}\right)\left[H\left(y^{\prime}, 0, x ; \bar{z}\right)-R\left(y^{\prime}, 0, x ; \bar{z}\right)\right]\right.\right. \\
& \left.\left.\quad-\delta\left(y_{3}+a\right)\left[H\left(y^{\prime},-a, x ; \bar{z}\right)-R\left(y^{\prime},-a, x ; \bar{z}\right)\right]\right\}\right) \\
& \quad+\chi_{+}\left(x_{3}\right) i \Lambda_{3}\left[\delta\left(y_{3}\right) T\left(y^{\prime}, 0, x ; \bar{z}\right)-\delta\left(y_{3}+a\right) T\left(y^{\prime},-a, x ; \bar{z}\right)\right] .
\end{aligned}
$$

COROLLARY 4.4. In the sense of $\mathscr{S}^{\prime}\left(\mathbf{R}_{y}^{3}\right)$,

$$
\begin{aligned}
& \Phi_{y}^{*} G_{+}(x, y ; z)(p)\left[\Lambda^{0}(p)-z I\right] \\
& =\chi_{+}\left(x_{3}\right)\left\{\frac{e^{i p x}}{(2 \pi)^{3 / 2}}+\frac{i E_{0}^{-1} \bar{t}}{\sqrt{2 \pi}}\left[\Phi_{2} I^{0}(\xi, 0, x ; \bar{z})-\Phi_{2} R^{0}(\xi, 0, x ; \bar{z})\right] E_{0} \Lambda_{3}^{0}\right\} \\
& \quad+\chi_{-}\left(x_{3}\right) i(2 \pi)^{-1 / 2} E^{-1} \bar{i}\left[\Phi_{2} T^{0}(\xi, 0, x ; \bar{z})\right] E_{0} \Lambda_{3}^{0} .
\end{aligned}
$$

Also,

$$
\begin{aligned}
\Phi_{y}^{*} G_{-} & (x, y ; z)(p)[\Lambda(p)-z I] \\
= & \chi_{-}\left(x_{3}\right)\left((2 \pi)^{-3 / 2} e^{i p x}\right. \\
& +i(2 \pi)^{-1 / 2} E^{-1}\left\{e^{-i a \eta} \bar{t}\left[\Phi_{2} H(\xi,-a, x ; \bar{z})-\Phi_{2} R(\xi,-a, x ; \bar{z})\right]\right. \\
& \left.\left.-i^{\bar{t}}\left[\Phi_{2} H(\xi, 0, x ; \bar{z})-\Phi_{2} R(\xi, 0, x ; \bar{z})\right]\right\}\right) E \Lambda_{3} \\
& +i(2 \pi)^{-1 / 2} \chi_{+}\left(x_{3}\right) E_{0}^{-1}\left\{e^{i a \rho} \bar{t}\left[\Phi_{2} T(\xi,-a, x ; \bar{z})-\Phi_{2} T(\xi, 0, x ; \bar{z})\right]\right\} E \Lambda_{3} .
\end{aligned}
$$

PROOF. Equation (4.49) is obtained from (4.47) by applying $\Phi_{y}$ to (4.47); take the transposed conjugate and use the relation (4.39). A similar operation gives (4.50).

\section{Representation of solutions by generalized eigenfunctions}

This section is rather computational in nature. There does not seem to be any way to avoid these details, but the impatient reader may refer to Theorem 5.14 below for the punch line.

Observe that the right-hand sides of (4.49) and (4.50) are known explicitly. We will express the resolvent operator in terms of these using certain density functions. In order to establish the relation between the resolvent operator and these density 
functions, let $\psi \in L_{2}\left(\mathbf{R}^{3}, \mathbf{C}^{6}\right), \psi_{ \pm}=\chi_{ \pm} \psi$ and $f \in \mathscr{K}$. Then using the Parseval identity for the Fourier transform $\left(\Phi=\Phi_{3}\right)$ we have

$$
\begin{aligned}
\left\langle\Phi_{y},\right. & \left.E_{0} \Phi_{\chi_{+}} G(\bar{z}) f\right\rangle \\
& =\left\langle\psi, E_{0} \Phi \chi_{+} G(\bar{z}) f\right\rangle=\left(\psi_{+}, G(\bar{z}) f\right)=\left(G(z) \psi_{+}, g\right) \\
& =\left(G(o, y ; z) \psi_{+}(y), f\right)=\left(\Phi_{y}^{*} G_{+}(\circ, y ; z)(p) \Phi \psi(p), f\right) \\
& =\sum_{j}\left(\Phi_{y}^{*} G_{+}(0, y ; z)(p)\left[\lambda_{j}^{0}(p)-z\right] P_{j}^{0}(p) E_{0}^{-1} E_{0} \Phi \psi(p),\left[\lambda_{j}^{0}(p)-\bar{z}\right]^{-1} f\right) \\
& =\sum_{j}\left(\Psi_{j}^{0}(\circ, p ; z) E_{0} \Phi \psi(p),\left[\lambda_{j}^{0}(p)-\bar{z}\right]^{-1} f\right) \\
& =\sum_{j}\left\langle\Phi \psi(p), E_{0}\left[\lambda_{j}^{0}(p)-\bar{z}\right]^{-1} \bar{i}_{j}^{0}(\circ, p ; z) E f\right\rangle .
\end{aligned}
$$

Therefore

$$
\begin{aligned}
\Phi_{\chi_{+}} G(\bar{z}) f(p) & =\sum_{j}\left[\lambda_{j}^{0}(p)-\bar{z}\right]^{-1} \int_{\mathbf{R}_{-a}^{3}}{ }^{\bar{T}} \Psi_{j}^{0}(x, p ; z) E(x) f(x) d x \\
& =\sum_{j}\left[\lambda_{j}^{0}(p)-\bar{z}\right]^{-1} \Psi_{j}^{0} f(p ; z),
\end{aligned}
$$

where

$$
\Psi_{j}^{0}(x, p ; z)=\Phi_{y}^{*} G_{+}(x, y ; z)(p)\left[\lambda_{j}^{0}(p)-z\right] P_{j}^{0}(p) E_{0}^{-1}
$$

and

$$
\Psi_{j}^{0} f(p ; z)=\int_{\mathbf{R}_{-a}^{3}}{ }^{i} \Psi_{j}^{0}(x, p ; z) E(x) f(x) d x, \quad j=0, \pm 1, \pm 2 .
$$

In exactly the same way,

$$
\begin{aligned}
\Phi_{\chi_{-} G(\bar{z}) f(p)} & =\sum_{j}\left[\lambda_{j}(p)-\bar{z}\right]^{-1} \int_{\mathbf{R}_{-a}^{3}}{ }^{\bar{r}} \Psi_{j}(x, p ; z) E(x) f(x) d x \\
& =\sum_{j}\left[\lambda_{j}(p)-\bar{z}\right]^{-1} \Psi_{j} f(p ; z),
\end{aligned}
$$

where

$$
\Psi_{j}(x, p ; z)=\Phi_{y}^{*} G_{-}(x, y ; z)(p)\left[\lambda_{j}(p)-z\right] P_{j}(p) E^{-1}
$$

and

$$
\Psi_{j} f(p ; z)=\int_{\mathbf{R}_{-a}^{3}}{ }^{i} \Psi_{j}(x, p ; z) E(x) f(x) d x
$$


The functions $\Psi_{j}^{0}(x, p ; z)$ and $\Psi_{j}(x, p ; z)$ are the density functions mentioned above.

Now (recall the inner product is defined with conjugation on the first place), for $z=\lambda+i \varepsilon$ we have from the resolvent identity and (5.1)-(5.6):

$$
\begin{aligned}
& ([G(\lambda+i \varepsilon)-G(\lambda-i \varepsilon)] f, f) \\
& =-2 i \varepsilon(G(\lambda \mp i \varepsilon) f, G(\lambda \mp i \varepsilon) f) \\
& =-2 i \varepsilon\left[\left\langle\chi_{+} G(\lambda \mp i \varepsilon) f, E_{0} \chi_{+} G(\lambda \mp i \varepsilon) f\right\rangle+\left\langle\chi_{-} G(\lambda \mp i \varepsilon) f, E_{\chi_{-}} G(\lambda \mp i \varepsilon) f\right\rangle\right] \\
& =-2 i \varepsilon\left[\left\langle\Phi \chi_{+} G(\lambda \mp i \varepsilon) f, E_{0} \Phi \chi_{+} G(\lambda \mp i \varepsilon) f\right\rangle\right. \\
& \left.\quad+\left\langle\Phi \chi_{-} G(\lambda \mp i \varepsilon) f, E \Phi \chi_{-} G(\lambda \mp i \varepsilon) f\right\rangle\right] \\
& =-2 i \sum_{j} \int_{\mathbf{R}^{3}}\left\{\frac{\varepsilon}{\left[\lambda_{j}^{0}(p)-\lambda\right]^{2}+\varepsilon^{2}}\left|\Psi_{j}^{0} f(p ; \lambda \pm i \varepsilon)\right|_{E_{0}}^{2}\right. \\
& \left.\quad+\frac{\varepsilon}{\left[\lambda_{j}(p)-\lambda\right]^{2}+\varepsilon^{2}}\left|\Psi_{j} f(p ; \lambda \pm i \varepsilon)\right|_{E}^{2}\right\} d p,
\end{aligned}
$$

where $|\cdot|_{E_{0}}$ and $|\cdot|_{E}$ denote the $E_{0}$ and $E$ norms in $\mathbf{C}^{6}$. Hence for any finite interval (see (4.2)) $(a, b) \subset \mathbf{R}$,

$$
\begin{aligned}
2^{-1}\{([F(b)+F(b-)] f, f)-([F(a)+F(a-)] f, f)\} \\
=\lim _{\varepsilon \downarrow 0}(2 \pi i)^{-1} \int_{a}^{b}([G(\lambda+i \varepsilon)-G(\lambda-i \varepsilon)] f, f) d \lambda \\
=\lim _{\varepsilon \downarrow 0} \pi^{-1} \int_{a}^{b} \sum_{j} \int_{\mathbf{R}^{3}}\left\{\frac{\varepsilon}{\left[\lambda_{j}^{0}(p)-\lambda\right]^{2}+\varepsilon^{2}}\left|\Psi_{j}^{0} f(p ; \lambda \pm i \varepsilon)\right|_{E_{0}}^{2}\right. \\
\left.\quad+\frac{\varepsilon}{\left[\lambda_{j}(p)-\lambda\right]^{2}+\varepsilon^{2}}\left|\Psi_{j} f(p ; \lambda \pm i \varepsilon)\right|_{E}^{2}\right\} d p d \lambda \\
=\lim _{\varepsilon \downarrow 0} \pi^{-1} \int_{\mathbf{R}^{3}} \sum_{j} \int_{a}^{b}\left\{\frac{\varepsilon}{\left[\lambda_{j}^{0}(p)-\lambda\right]^{2}+\varepsilon^{2}}\left|\Psi_{j}^{0} f(p ; \lambda \pm i \varepsilon)\right|_{E_{0}}^{2}\right. \\
\left.\quad+\frac{\varepsilon}{\left[\lambda_{j}(p)-\lambda\right]^{2}+\varepsilon^{2}}\left|\Psi_{j} f(p ; \lambda \pm i \varepsilon)\right|_{E}^{2}\right\} d \lambda d p .
\end{aligned}
$$

By (5.1) and (5.4), $\sum_{j}\left[\lambda_{i}(p)-\bar{z}\right]^{-1} \Psi_{j} f(p ; z)$ and $\sum_{j}\left[\lambda_{i}^{0}(p)-\bar{z}\right]^{-1} \Psi_{j}^{0} f(p ; z)$ are continuous $L_{2}$-valued functions of $z$ for $\operatorname{Im}(z) \neq 0$. To obtain the desired representation of the spectral family, it thus remains to pass to the limit under the integral sign in (5.7). The justification for this is based on explicit formulae for the functions $\Psi_{j} f(p ; \lambda \pm i \varepsilon), \Psi_{j}^{0} f(p ; \lambda \pm i \varepsilon)$ and their limits obtained by passing to the limit $\varepsilon \downarrow 0$. Except for the Alfven waves (see (5.29)-(5.31) below), we can write these functions 
as

$$
\begin{aligned}
\Psi_{j}^{0}(x, p ; z)= & \chi_{+}\left(x_{3}\right)\left\{(2 \pi)^{-3 / 2} e^{i p x} P_{j}^{0}(p) E_{0}^{-1}+i(2 \pi)^{-1 / 2} E_{0}^{-1}\right. \\
& \left.\times{ }^{\bar{\imath}}\left[\Phi_{2} I^{0}(\xi, 0, x ; \bar{z})-\Phi_{2} R^{0}(\xi, 0, x ; \bar{z})\right] E_{0} \Lambda_{3}^{0} P_{j}^{0}(p) E_{0}^{-1}\right\} \\
& +\chi_{-}\left(x_{3}\right) i(2 \pi)^{-1 / 2} E^{-1} \bar{\imath}\left[\Phi_{2} T^{0}(\xi, 0, x ; \bar{z})\right] E_{0} \Lambda_{3}^{0} P_{j}^{0}(p) E_{0}^{-1}, \\
\Psi_{j}(x, p ; z)= & \chi_{-}\left(x_{3}\right)\left((2 \pi)^{-3 / 2} e^{i p x} P_{j}(p) E^{-1}\right. \\
& +i(2 \pi)^{-1 / 2} E^{-1}\left\{e^{i a \eta} \bar{i}\left(\Phi_{2} H(\xi,-a, x ; \bar{z})-\Phi_{2} R(\xi,-a, x ; \bar{z})\right)\right\} \\
& \left.-{ }^{\bar{\imath}}\left[\Phi_{2} H(\xi, 0, x ; \bar{z})-\Phi_{2} R(\xi, 0, x ; \bar{z})\right] E \Lambda_{3} P_{j}(p) E^{-1}\right) \\
= & \chi_{+}\left(x_{3}\right) i(2 \pi)^{-1 / 2} E_{0}^{-1}\left\{e^{i a \eta} \bar{t}\left[\Phi_{2} T(\xi,-a, x ; \bar{z})\right]\right. \\
& \left.-{ }^{\bar{t}}\left[\Phi_{2} T(\xi, 0, x ; \bar{z})\right]\right\} E \Lambda_{3} P_{j}(p) E^{-1} .
\end{aligned}
$$

We need the following lemma which is proved by direct observation of the relevant quantities.

LEMMA 5.1. With $P(\xi, z, \pm \tau), P^{0}\left(\xi, z, \pm \tau^{0}\right)$ and $P_{j}(p), P_{j}^{0}(p)$ as previously defined (see (2.8)),

$$
\begin{aligned}
P(\xi, z, \pm \tau) \Lambda_{3} P_{j}(p) & =(\eta \mp \tau)^{-1}\left[\lambda_{j}(p)-z\right] P(\xi, z, \pm \tau) P_{j}(p) \\
P^{0}\left(\xi, z, \pm \tau^{0}\right) \Lambda_{3}^{0} P_{j}^{0}(p) & =\left(\eta \mp \tau^{0}\right)^{-1}\left[\lambda_{j}^{0}(p)-z\right] P^{0}\left(\xi, z, \pm \tau^{0}\right) P_{j}^{0}(p) .
\end{aligned}
$$

Using the forms of $H, R, T, e t c .,(5.8)$ and (5.9), we now have

$$
\begin{aligned}
\Psi_{j}^{0}(x, p ; z)= & \frac{\chi_{+}\left(x_{3}\right)}{(2 \pi)^{3 / 2}}\left\{e^{i x p} I+\frac{e^{i x^{\prime} \xi} e^{i \tau^{0} x_{3}} z}{c_{0}^{2} \tau^{0}}\left[\frac{\lambda_{j}^{0}(p)-z}{\eta+\tau^{0}} C^{0}(\xi, z) P^{0}\left(\xi, z,-\tau^{0}\right)\right.\right. \\
& \left.\left.-\frac{\lambda_{j}^{0}(p)-z}{\eta-\tau^{0}} P^{0}\left(\xi, z, \tau^{0}\right)\right]\right\} P_{j}^{0}(p) E_{0}^{-1}-\frac{\chi_{-}\left(x_{3}\right) e^{i x^{\prime} \xi} z}{(2 \pi)^{3 / 2} \tau^{0} c_{0}^{2}} \\
& \times Q\left(\tau\left(x_{3}+a\right)\right) D^{0^{\prime}}(x, z) \frac{\lambda_{j}^{0}(p)-z}{\eta+\tau^{0}} P^{0}\left(\xi, z,-\tau^{0}\right) P_{j}^{0}(p) E_{0}^{-1}, \quad(5.11) \\
\Psi_{j}(x, p ; z)= & \frac{\chi-\left(x_{3}\right)}{(2 \pi)^{3 / 2}}\left(e^{i x p} I-\frac{e^{i x^{\prime} \xi} e^{i \tau x_{3}} z}{c^{2} \tau}\left\{e ^ { i a \eta } e ^ { i \tau x _ { 3 } } P ( x , z , \tau ) \left[\frac{\lambda_{j}(p)-z}{\eta-\tau} I\right.\right.\right. \\
& \left.\left.\left.-\frac{\lambda_{j}(p)-z}{\eta+\tau} Q_{a}\right]-Q\left(\tau\left(x_{3}+a\right)\right) P(\xi, z,-\tau) \frac{\lambda_{j}(p)-z}{\eta+\tau}\right\}\right) P_{j}(p) E^{-1} \\
& +\frac{e^{i x^{\prime} \xi} z\left(\lambda_{j}(p)-z\right)}{(2 \pi)^{3 / 2} c^{2}}\left[-\chi-\left(x_{3}\right) \frac{e^{i a \tau}}{\tau} Q\left(\tau\left(x_{3}+a\right)\right) C^{\prime}(\xi, z)\right. \\
& \left.+\chi_{+}\left(x_{3}\right) \frac{e^{i \tau^{0} x_{3}}}{\tau} E_{0}^{-1} D^{\prime}(\xi, z) E\right] P(\xi, z, \tau) W(p ; z) P_{j}(p) E^{-1}, \quad \text { (5.12) }
\end{aligned}
$$


[19]

$$
W(p ; z)=\frac{e^{-i a \tau}-e^{-i a \eta}}{\eta-\tau}+\frac{e^{-i a \eta}-e^{i a \tau}}{\eta+\tau} Q_{a} .
$$

The plane-wave modes are obtained by passing to the limits $z \rightarrow \lambda_{j 2}^{0}(p) \pm i 0=$ $j c_{0}|p| \pm i 0, z \rightarrow \lambda_{j 2}(p) \pm i 0 \approx j c|p| \pm i 0, j= \pm 1$, in (5.11) and (5.12). In so doing, it is convenient to observe the following facts:

$$
\begin{aligned}
& \tau\left(\xi, \lambda_{j 2}(p) \pm i 0\right)=\tau^{0}\left(\xi, \lambda_{j 2}^{0}(p) \pm i 0\right)= \pm j|\eta|, \\
& \frac{\lambda_{j 2}(p)-z}{\eta-\tau}\left(\lambda_{j 2}(p) \pm i 0\right)=\frac{j c \eta}{|p|} \chi_{\mathbf{R}_{ \pm j}}(\eta), \\
& \frac{\lambda_{j 2}(p)-z}{\eta+\tau}\left(\lambda_{j 2}(p) \pm i 0\right)=\frac{j c \eta}{|p|} \chi_{\mathbf{R}_{\mp j}}(\eta), \\
& \frac{\lambda_{j 2}^{0}(p)-z}{\eta-\tau^{0}}\left(\lambda_{j 2}^{0}(p) \pm i 0\right)=\frac{j c 0 \eta}{|p|} \chi_{\mathbf{R}_{ \pm j}}(\eta), \\
& \frac{\lambda_{j 2}^{0}(p)-z}{\eta+\tau^{0}}\left(\lambda_{j 2}^{0}(p) \pm i 0\right)=\frac{j c_{0} \eta}{|p|} \chi_{\mathbf{R}_{\mp j}}(\eta), \\
& |\eta| \chi_{\mathbf{R}_{\mp i}}(\eta)=\mp j \eta, \quad|\eta| \chi_{\mathbf{R}_{ \pm j}}(\eta)= \pm j \eta .
\end{aligned}
$$

From the first and last equalities it follows that

$$
\tau\left(\xi, \lambda_{j 2}(p) \pm i 0\right) \chi_{\mathbf{R}_{ \pm j}}(\eta)=\eta \quad \text { and } \quad \tau\left(\xi, \lambda_{j 2}(p) \pm i 0\right) \chi_{\mathbf{R}_{\neq j}}(\eta)=-\eta
$$

Hence from (5.12) and (5.13),

$$
\begin{aligned}
& {\left[\lambda_{j 2}(p)-z\right] W(p ; z)\left(\lambda_{j 2}(p) \pm i 0\right)} \\
& \quad=\frac{j c \eta}{|\boldsymbol{p}|}\left[\left(e^{-i a \eta}-e^{-i a \eta}\right) \chi_{\mathbf{R}_{ \pm j}}(\eta)+\left(e^{-i a \eta}-e^{-i a \eta}\right) \chi_{\mathbf{R}_{\mp j}}(\eta)\right]=0
\end{aligned}
$$

so that

$$
\begin{aligned}
\Psi_{j 2}\left(x, p ; \lambda_{j 2}(p) \pm i 0\right) \\
=\frac{\chi_{+}\left(x_{3}\right)}{(2 \pi)^{3 / 2}}\left\{e ^ { i x \eta } \mp j \operatorname { s g n } \eta e ^ { i x ^ { \prime } \xi } e ^ { \pm j a | \eta | } \left[e^{-i a \eta} e^{i \eta x_{3}} P_{j 2}(p) \chi_{\mathbf{R}_{ \pm x}}(\eta)\right.\right. \\
\left.\left.\quad-\left(e^{-i a \eta} e^{-x_{3} \eta} P_{j 2}(x,-\eta) Q_{a}+Q\left(-\eta\left(x_{3}+a\right)\right) P_{j 2}(p)\right) \chi_{\mathbf{R}_{\mp j}}(\eta)\right]\right\} P_{j}(p) E^{-1} .
\end{aligned}
$$

Now $Q\left(-\eta\left(x_{3}+a\right)\right)=\exp \left(i \eta\left(x_{3}+a\right)\right) I-\exp \left(-i \eta\left(x_{3}+a\right)\right) Q_{a}$ and $Q_{a} P_{j}(p)=$ $P_{j}(\xi,-\eta) Q_{a}$. Hence

$$
\begin{aligned}
& \Psi_{j 2}\left(x, p ; \lambda_{j 2}(p) \pm i 0\right) \\
& \quad=\frac{\chi_{+}\left(x_{3}\right)}{(2 \pi)^{3 / 2}}\left\{e^{i x p}-e^{i x p} \chi_{\mathbf{R}_{ \pm j}}(\eta)-e^{-2 i a \eta} e^{i x^{\prime} \xi} e^{-i x_{3} \eta} Q_{a} \chi_{\mathbf{R}_{\mp j}}(\eta)\right.
\end{aligned}
$$




$$
\left.-e^{i x p} \chi_{\mathbf{R}_{\mp j}}(\eta)+e^{-2 i a \eta} e^{i x^{\prime} \xi} e^{-i x_{3} \eta} Q_{a} \chi_{\mathbf{R}_{F j}}(\eta)\right\} P_{j 2}(p) E^{-1}=0 .
$$

Hence, no plane-wave modes with the phase speed $c$ of the layer exist. Thus the functions $\Psi_{j}(x, p ; z)$ contribute only to trapped modes of the layer $\mathbf{R}^{2} \times(-a, 0)$.

Consideration is now given to the limits $\Psi_{j}^{0}\left(x, p ; \lambda_{j}^{0}(p) \pm i 0\right)$. We define

$$
n=c_{0} c^{-1}, \quad \theta^{\prime}=n^{-1} \omega^{\prime}, \quad \omega \in \mathbf{R}^{3} \text { and }|\omega|=1 \text { (recall } \sqrt{\rho \mu}=c^{-1} \text { ). }
$$

Let

$$
\theta_{3}=\left\{\begin{aligned}
-\sqrt{1-\left|\theta^{\prime}\right|^{2}}, & \text { if } n>1, \text { or } n<1 \text { and } \omega_{3}^{2}>\left(n^{-2}-1\right)\left|\omega^{\prime}\right|^{2} \\
0, & \text { if } n<1 \text { and } \omega_{3}^{2}=\left(n^{-2}-1\right)\left|\omega^{\prime}\right|^{2} \\
-i \sqrt{\left|\theta^{\prime}\right|^{2}-1}, & \text { if } n<1 \text { and } \omega_{3}^{2}<\left(n^{-2}-1\right)\left|\omega^{\prime}\right|^{2}
\end{aligned}\right.
$$

and $\chi=\left(\chi_{1}, \chi_{2}, \chi_{3}\right)=n|p|\left(\theta_{1}, \theta_{2}, \theta_{3}\right)$, so that $|\chi|=n|p|$. Then

$$
\tau\left(\xi, \lambda_{j 2}^{0}(p) \pm i 0\right)=\left\{\begin{aligned}
\mp j \chi_{3}, & \text { if } n>1, \text { or } n<1 \text { and } \\
& \omega_{3}^{2}>\left(n^{-2}-1\right)\left|\omega^{\prime}\right|^{2}, \\
-\chi_{3}=-n|p| \theta_{3} & \\
=i n|p|\left|\theta_{3}\right|, & \text { if } n<1 \text { and } \omega_{3}^{2}<\left(n^{-2}-1\right)\left|\omega^{\prime}\right|^{2} .
\end{aligned}\right.
$$

The third term on the right of (5.11) gives $-\chi_{\mathbf{R}_{ \pm j}}(\eta) e^{i x p} P_{j}(p) E_{0}^{-1}$ in the limit as $z \rightarrow \lambda_{j 2}^{0}(p) \pm i 0$ and thus

$$
\Psi_{j 2}^{0}\left(x, p ; \lambda_{j 2}^{0}(p) \pm i 0\right) \equiv \Psi_{j 2}^{0 \pm}(x, p)=-\chi_{\mathbf{R}_{ \pm j}}(\eta) \Psi_{j 2}^{0 \pm}(x, p), \quad j= \pm 1 .
$$

We can use this to compute the reflection and transmission coefficients in (5.11). We define $\tilde{p}=\left(p_{1}, p_{2},-p_{3}\right)$, then

$$
\begin{aligned}
\Psi_{j 2}^{0}(x, p ; & \left.\lambda_{j 2}^{0}(p) \pm i 0\right) \equiv \Psi_{j 2}^{0 \pm}(x, p) \\
= & \lim _{z \rightarrow \lambda_{j 2}^{0}(p) \pm i 0} \frac{\chi_{+}\left(x_{3}\right)}{(2 \pi)^{3 / 2}}\left\{e^{i x p} I+\frac{e^{i x^{\prime} \xi} e^{i \tau^{0} x_{3}} z}{c_{0}^{2} \tau^{0}}\left[\frac{\lambda_{j}^{0}(p)-z}{\eta+\tau^{0}} C^{0}(\xi, z) P^{0}\left(\xi, z,-\tau^{0}\right)\right.\right. \\
& \left.\left.-\frac{\lambda_{j}^{0}(p)-z}{\eta-\tau^{0}} P^{0}\left(\xi, z, \tau^{0}\right)\right]\right\} P_{j 2}^{0}(p) E_{0}^{-1} \\
& -\frac{\chi_{-}\left(x_{3}\right) e^{i x^{\prime} \xi} z}{(2 \pi)^{3 / 2} c_{0}^{2} \tau^{0}} Q\left(\tau\left(x_{3}+a\right)\right) D^{0^{\prime}}(\xi, z) \frac{\lambda_{j}^{0}(p)-z}{\eta+\tau^{0}} P^{0}\left(\xi, z,-\tau^{0}\right) P_{j 2}^{0}(p) E_{0}^{-1} \\
= & (2 \pi)^{-3 / 2} \chi_{\mathbf{R}_{\mp j}}(\eta)\left\{\chi_{+}\left(x_{3}\right)\left[e^{i x p} I-E^{i x \tilde{p}} C^{0}(p)\right] P_{j 2}^{0}(p)\right. \\
& \left.+\chi_{-}\left(x_{3}\right) e^{i x^{\prime} \xi} Q^{0}\left(\chi_{3}\left(x_{3}+a\right)\right) P_{j 2}^{0}(\theta) D^{0}(p)\right\} E_{0}^{-1} \\
= & (2 \pi)^{-3 / 2} \chi_{\mathbf{R}_{\mp j}}(\eta)\left\{\chi_{+}\left(x_{3}\right)\left[e^{i x p} I-e^{i x \bar{p}} C^{0}(p)\right]\right.
\end{aligned}
$$




$$
\begin{aligned}
& \left.+\chi_{-}\left(x_{3}\right) c^{-2} c_{0}^{2} e^{i x^{\prime} \xi} Q^{0}\left(\chi_{3}\left(x_{3}+a\right)\right) D(p)\right\} P_{j 2}(p) E_{0}^{-1} \\
= & (2 \pi)^{-3 / 2} \chi_{\mathbf{R}_{\mp j}}(\eta)\left\{\chi_{+}\left(x_{3}\right)\left[e^{i x p} P_{j 2}^{0}(p)-e^{i x \tilde{p}} P_{j 2}^{0}(\tilde{p}) C^{0}(p)\right]\right. \\
& \left.+\chi_{-}\left(x_{3}\right) c^{-2} c_{0}^{2}\left[e^{i x \chi} e^{i a x_{3}} P_{j 2}(\theta)-e^{i x \tilde{x}} e^{-i a x_{3}} P_{j 2}(\tilde{\theta})\right] Q_{a}\right\} E_{0}^{-1}
\end{aligned}
$$

These formulae are valid for $n>1$ or $n<1$. The first formula is based on the matrices $C^{0}(p), D^{0}(p)$ and $D(p)$ given in Appendix II and (5.14). The matrices $C^{0}(p), D^{0}(p)$ and $D(p)$ being derived from $C^{0}(\xi, z), D^{0^{\prime}}(\xi, z)$ and $D^{\prime}(\xi, z)$ also satisfy the following relations which can be verified directly without difficulty from the corresponding formulae found in Appendix II:

$$
\begin{gathered}
Q_{a} P_{j 2}(\omega)=P_{j 2}(\tilde{\omega}) Q_{a}, \quad Q_{a} P_{j 2}^{0}(\omega)=P_{j 2}^{0}(\tilde{\omega}) Q_{a}, \\
C^{0}(p) P_{j 2}^{0}(\omega)=P_{j 2}^{0}(\tilde{\omega}) C^{0}(p), \\
c_{0}^{-2} D^{0}(p) P_{j 2}^{0}(\omega)=c^{-2} P_{j 2}(\theta) D(p), \\
L\left[C^{0}(p)+Q^{0}\left(a \chi_{3}\right) D^{0}(p)\right]=L, \\
{\left[\rho_{0} C^{0}(p)+\rho Q^{0}\left(a \chi_{3}\right) D^{0}(p)\right]_{33}=\rho_{0},} \\
{\left[\mu_{0} C^{0}(p)+\mu Q^{0}\left(a \chi_{3}\right) D^{0}(p)\right]_{33}=\mu_{0},} \\
{ }^{i} C^{0}(p)=C^{0}(\tilde{p}), \\
{ }^{\bar{t}}\left[Q^{0}\left(\chi_{3}\left(x_{3}+a\right)\right) D^{0}(p)\right]=Q^{0}\left(\chi_{3}\left(x_{3}+a\right)\right) D^{0}(\tilde{p}), \\
{ }^{i}\left[Q^{0}\left(\chi_{3}\left(x_{3}+a\right)\right) D(p)\right]=Q^{0}\left(\chi_{3}\left(x_{3}+a\right)\right) D(\tilde{p}) .
\end{gathered}
$$

From (5.16) and the fact $(j= \pm 1)$

$$
\Lambda(D) e^{i x x} P_{j 2}(\theta)=n|p| e^{i x x} \Lambda(\theta) P_{j 2}(\theta)=j c n|p| e^{i x x} P_{j 2}(\theta)=\lambda_{j 2}^{0}(p) e^{i x x} P_{j 2}(\theta)
$$

we infer that $\Psi_{j 2}^{0 \pm}(x, p)$ satisfies the equation

$$
\Lambda^{\prime}(D) \Psi_{j 2}^{0 \pm}(x, p)=\lambda_{j 2}^{0} \Psi_{j 2}^{0 \pm}(x, p), \quad 0 \neq x_{3} \in(-a, 0) .
$$

From (5.16) and the definition of $Q^{0}$ from Appendix II it is clear that $\Psi_{j 2}^{0 \pm}(x, p)$ satisfies the boundary conditions

$$
\mathfrak{B} \Psi_{j 2}^{0 \pm}\left(x^{\prime},-a, p\right)=0 \quad \text { and also } \quad \Psi_{j 2}^{0 \pm}\left(x^{\prime},-a, p\right)_{6 i}=0 .
$$

The equations (5.21) and (5.16) further imply that

$$
L \Psi_{j 2}^{0 \pm}\left(x^{\prime}, 0-, p\right)=L \Psi_{j 2}^{0 \pm}\left(x^{\prime}, 0+, p\right)
$$

From (5.21) and (5.22) we also see that (3.8) is satisfied. Equation (5.16) also immediately shows that the entries of the last three rows form six divergence-free vectors, 
and furthermore, the second row consists entirely of zeroes. Thus superpositions of $\Psi_{j 2}^{0 \pm}(x, p)$ on $p$ which are square integrable satisfy the conditions of Proposition 3.10 for residence in the complement of the null space of $\Lambda^{\prime}$ in $\mathscr{K}$. Hence these solutions do propagate.

Up to now, we have not examined the Alfven modes. They arise from consideration of the speeds $\lambda_{ \pm 1}(p)$ and the projectors $P_{ \pm 1}(p)$. As will be seen, they have a trivial structure and do not couple with the other modes at the boundary. Note that these modes must automatically satisfy the boundary condition (!) (see (2.9)).

Since Alfven waves propagate parallel to the layer, there is no reflection from the boundaries. Therefore we have $(j= \pm 1)$

$$
\begin{aligned}
\Psi_{j}^{ \pm}(x, p) & =\chi_{-}\left(x_{3}\right) e^{i x p} P_{j 1}(p) E^{-1}, \\
\Psi_{j}^{0 \pm}(x, p) & =\chi_{+}\left(x_{3}\right) e^{i x p} P_{j 1}^{0}(p) \Pi^{0} E_{0}^{-1}, \\
\Lambda^{\prime}(D) \Psi_{j}^{ \pm}(x, p) & =e^{i x p} \Lambda(p) P_{j 1}(p) E^{-1}=\lambda_{j}(p) \Psi_{j}^{ \pm}(x, p),
\end{aligned}
$$

where $\Psi_{j}^{ \pm}(x, p)$ satisfies the boundary condition. ( $\Pi^{0}$ is the diagonal matrix $\operatorname{diag}\left(\rho_{0} / \rho, A, \rho_{0} / \rho, 1, A, 1\right)$.) Furthermore, $L \Psi_{j}^{ \pm}\left(x^{\prime}, 0-, p\right)=L \Psi_{j}^{0 \pm}\left(x^{\prime}, 0+, p\right)$. Also, the six columns formed by the last three rows of $\Psi_{j}^{ \pm}(x, p)$ and $\Psi_{j}^{0 \pm}(x, p)$ are divergence-free vectors and the second coordinate of each column is zero. From this we see that superpositions of these functions which are square integrable lie in the complement of the null space of $\Lambda^{\prime}$ in $\mathscr{K}$.

The case the $n<1(5.14)$ and $\omega^{2}<\left(n^{-2}-1\right)\left|\omega^{\prime}\right|^{2}$ corresponds to what would be total reflection at $x_{3}=0$ if there were no boundary at $x_{3}=-a$. The first term of $\chi_{-}\left(x_{3}\right) \Psi_{j 2}^{0 \pm}(x, p)$ in (5.16) corresponds to a transmitted wave and the exponential factor in $x_{3}$ decays from $x_{3}=0$ to $x_{3}=-a$. Now, however, there is a wave reflected at $x_{3}=-a$ corresponding to the second term of $\chi_{-}\left(x_{3}\right) \Psi_{j 2}^{0 \pm}(x, p)$ with exponential factor which increases from $x_{3}=0$ to $x_{3}=-a$.

We now need the following somewhat startling lemma.

LEMMA 5.2. $J\left(\xi, z_{1}\right)=0$ if and only if $K\left(\xi, z_{1}\right)=0$. The zeroes of $K$ (and hence J) are simple.

(Proof given in Appendix I.)

In the case $n>1$, further contribution to the limit in (5.7) comes from the singularities of the reflection and transmission coefficients in the density functions (5.11) and (5.12), that is, from the roots $v_{j k}$ of $J$ and $K$ (Theorem 4.1, Lemma 5.2). Let $j \pm 1$ and let $O^{\circ}(1)$ and $O(1)$ be the terms from (5.11) and (5.12) not containing $C^{0}, D^{0 \prime}$, $C^{\prime}$ and $D^{\prime}$. Define the trapped modes as

$$
\Sigma^{0}\left(x, p ; z ; v_{j k}\right)=\sum_{\ell} \frac{z-v_{j k}}{\lambda_{\ell}^{0}-z} \psi_{\ell}^{0}(x, p ; z)
$$




$$
\begin{aligned}
&= \frac{z e^{i x^{\prime} \xi}}{(2 \pi)^{3 / 2} \tau^{0}} \frac{z-v_{j k}}{\eta+\tau^{0}}\left[\chi_{+}\left(x_{3}\right) e^{i \tau^{0} x_{3}} C^{0}(\xi, z)\right. \\
&\left.-\chi_{-}\left(x_{3}\right) Q\left(\tau^{0}\left(x_{3}+a\right)\right) D^{0 \prime}(\xi, z)\right] c_{0}^{-2} P^{0}\left(\xi, z,-\tau^{0}\right) E_{0}^{-1}+O^{0}(1) \\
& \Sigma\left(x, p ; z ; v_{j k}\right)=\sum_{\ell} \frac{z-v_{j k}}{\lambda_{\ell}-z} \psi_{\ell}(x, p ; z) \\
&= \\
& \quad \frac{z\left(z-v_{j k}\right)}{(2 \pi)^{3 / 2}}\left[-\chi_{-}\left(x_{3}\right) \tau^{-1} e^{i \tau a} Q\left(\tau\left(x_{3}+a\right)\right) C^{\prime}(\xi, z)\right. \\
&\left.\quad+\chi_{+}\left(x_{3}\right) \tau^{0^{-1}} e^{i \tau^{0} x_{3}} E_{0}^{-1} D^{\prime}(\xi, z) E\right] c^{-2} P(\xi, z, \tau) W(p, z) E^{-1}+O(1) .
\end{aligned}
$$

Also define the matrices

$$
\begin{aligned}
C^{0}\left(\xi, v_{j k}\right)= & \left(z-v_{j k}\right) C^{0}(\xi, z), \quad D^{ \pm \ni}\left(\xi, v_{j k}\right)=\left(z-v_{j k}\right) D^{\prime}(\xi, z), \\
D^{0}\left(\xi, v_{j k}\right)= & \left(z-v_{j k}\right) D^{0 \prime}(\xi, z) \quad \text { and } \quad C^{ \pm \prime}\left(\xi, v_{j k}\right)=\left(z-v_{j k}\right) C^{\prime}(\xi, z), \\
& D^{ \pm}\left(\xi, v_{j k}\right)=\left(i \tau_{j k}^{0}\right)^{-1} E_{0}^{-1} D^{ \pm \prime}\left(\xi, v_{j k}\right) E \quad \text { and } \\
& C^{ \pm}\left(\xi, v_{j k}\right)=-Q(\mp j) E^{i a \tau_{j k}} \tau_{j k}^{-1} C^{ \pm \prime}\left(\xi, v_{j k}\right) .
\end{aligned}
$$

Passing to the limit in (4.42)-(4.46) gives important relations for these matrices. Passing to the limit in (5.32)-(5.33) gives

$$
\begin{aligned}
& \Sigma^{0}\left(x, p ; v_{j k} \pm i 0\right) \\
& =-\frac{i j \chi_{r_{k}}(|\xi|) v_{k} e^{i x^{\prime} \xi}}{(2 \pi)^{3 / 2} \tau_{k}^{0}}\left[\chi_{+}\left(x_{3}\right) e^{i \tau_{k}^{0} x_{3}} C^{0}\left(\xi, v_{j k}\right)\right. \\
& \left.\quad+\chi_{-}\left(x_{3}\right) Q^{0}\left(\tau_{k}\left(x_{3}+a\right)\right) D^{0}\left(\xi, v_{j k}\right)\right] \frac{P^{0}\left(\xi, j v_{k},-i \tau_{k}^{0}\right) E_{0}^{-1}}{c_{0}^{2}\left(\eta+i \tau_{k}^{0}\right)} \\
& \Sigma^{ \pm}\left(x, p ; z ; v_{j k} \pm i 0\right) \\
& \quad=\frac{j \chi_{r_{k}}(|\xi|) v_{k} e^{i x^{\prime} \xi}}{(2 \pi)^{3 / 2}}\left[\chi_{+}\left(x_{3}\right) e^{i i_{k}^{0} x_{3}} D^{ \pm}\left(\xi, v_{j k}\right)\right. \\
& \left.\quad+\chi_{-}\left(x_{3}\right) Q^{0}\left(\tau_{k}\left(x_{3}+a\right)\right) C^{ \pm}\left(\xi, v_{j k}\right)\right] \frac{P\left(\xi, j v_{k},-j \tau_{k}\right) E_{0}^{-1}}{c^{2}\left(\eta+i \tau_{k}^{0}\right)} W^{ \pm}\left(p ; v_{j k}\right)
\end{aligned}
$$

and

$$
W^{ \pm}\left(p ; v_{j k}\right) \equiv W\left(p ; v_{j k} \pm i 0\right)=\frac{e^{\mp i a j \tau_{k}}-e^{i a \eta}}{\eta \mp j \tau_{k}} i+\frac{e^{-i a \eta}-e^{ \pm i a j \tau_{k}}}{\eta \pm j \tau_{k}} Q_{a} .
$$

The term $\chi_{r_{k}}$ is the characteristic function on the interval $\left(r_{k}, \infty\right)$. Note that these two waves decay exponentially away from the layer. They are true trapped waves and not surface waves. This agrees with the results of [6] for the more complex system studied there. 
Let

$$
\begin{aligned}
\Sigma^{0}\left(x, \xi ; v_{j k} \pm i 0\right) & =\left(\eta+i \tau_{k}^{0}\right) \Sigma^{0}\left(x, p ; v_{j k} \pm i 0\right) \quad \text { and } \\
\Sigma^{\sim}\left(x, \xi ; z ; v_{j k} \pm i 0\right) & =\Sigma^{ \pm}\left(x, p ; z ; v_{j k} \pm i 0\right)\left[W^{ \pm}\left(p ; v_{j k}\right)\right]^{-1} Q( \pm j) .
\end{aligned}
$$

We note the following relation, useful in verifying the properties of the functions (5.37)-(5.38):

$$
\Lambda\left(\xi,-\tau_{k}\right) Q_{a}=Q_{a} \Lambda\left(\xi, \tau_{k}\right)
$$

Using the forms of $C^{ \pm}\left(\xi, v_{j k}\right), D^{ \pm}\left(\xi, v_{j k}\right), C^{0}\left(\xi, v_{j k}\right)$ and $D^{0}\left(\xi, v_{j k}\right)$ given in Appendix II, it is a somewhat tedious but straightforward problem to check the identities $\left(x_{3} \neq 0\right)$

$$
\begin{aligned}
\Lambda^{\prime}(D) \Sigma^{0}\left(x, \xi ; v_{j k} \pm i 0\right) & =j v_{k}(\xi) \Sigma^{0}\left(x, \xi ; v_{j k} \pm i 0\right) \quad \text { and } \\
\Lambda^{\prime}(D) \Sigma^{\sim}\left(x, \xi ; z ; v_{j k} \pm i 0\right) & =j v_{k}(\xi) \Sigma^{\sim}\left(x, \xi ; z ; v_{j k} \pm i 0\right)
\end{aligned}
$$

From the definition of $Q^{0}$ (see Appendix II), $\Sigma^{\sim}$ and $\Sigma^{0}$ satisfy the boundary condition. The continuity and jump conditions (3.6) and (3.8) are satisfied and the last three rows form divergence-free vectors as columns $\left(x_{3} \neq 0\right)$. Hence superpositions of these functions on $x$ which are square integrable belong to $\overline{\mathscr{K}}$. Observation of the functions in Appendix II and the proof of Theorem 4.1 (given in Appendix I) shows that, away from the neighborhood of $\beta$, the denominators of all terms in (5.35)-(5.36), (5.37)-(5.38) are bounded away from zero.

Now suppose that $g \in \mathscr{D}\left(\mathbf{R}_{-a}^{3}\right)$ and set $g_{+}=\chi_{+} g, g_{a}=\chi_{(0, a)}\left(x_{3}\right) g\left(x^{\prime}, x_{3},-a\right)$ and $\tilde{p}=(\xi,-\eta)$. Define

$$
\begin{aligned}
\Psi_{j 2}^{0} g(p)= & \int_{\mathbf{R}_{-a}^{3}} \bar{i}_{j 2}^{0 \pm}(x, p) E(x) g(x) d x \\
= & \frac{\chi_{\mathbf{R}_{\mp j}}(\eta) P_{j 2}^{0}(\omega)}{(2 \pi)^{3 / 2}}\left\{\int_{\mathbf{R}_{+}^{3}}\left[e^{-i x_{3} \eta}-C^{0}(\tilde{p}) e^{i x_{3} \eta}\right] \Phi_{2} g\left(\xi, x_{3}\right) d x_{3}\right. \\
& \left.+E_{0}^{-1} D^{0}(\tilde{p}) E \int_{-a}^{0} Q^{0}\left(x_{3}+a\right) \Phi_{2} g\left(\xi, x_{3}\right) d x_{3}\right\} \\
= & \chi_{\mathbf{R}_{\mp j}}(\eta) P_{j 2}^{0}(\omega)\left\{\Phi_{3} g_{+}(p)-C^{0}(\tilde{p}) \Phi_{3} g_{+}(\tilde{p})\right. \\
& \left.+D^{0}(\tilde{p}) E_{0}^{-1} E\left[\Phi_{3} g_{a}\left(\xi,-x_{3}\right)-Q_{a} \Phi_{3} g_{a}\left(\xi, x_{3}\right)\right]\right\} .
\end{aligned}
$$

LEMMA 5.3. For $g \in \mathscr{D}\left(\mathbf{R}_{-a}^{3}\right), \Psi_{j 2}^{0} g(p) \in \mathscr{K}^{0}\left(\mathscr{K}^{0}\right.$ means $L_{2}$ with the $E_{0}$ inner product).

The proof is a straightforward application of the definitions. 
The following pertains to the case $n>1$. See (5.14) for the definition of $n$. This is the more interesting case where trapped waves exist; $n<1$ is the same except that the trapped mode terms are absent. For $g \in \mathscr{D}\left(\mathbf{R}_{-a}^{3}\right), j= \pm 1, k \in \mathbf{Z}_{+}$, recalling (5.35)-(5.41), we define

$$
\begin{aligned}
\Sigma^{0}\left(v_{j k}\right) g(p) & =\int_{\mathbf{R}_{+}^{3}}{ }^{i} \Sigma^{0}\left(x, p ; v_{j k}\right) E(x) g(x) d x \\
& =\left[\eta-i \tau_{j k}^{0}(\xi)\right]^{-1} \int_{\mathbf{R}_{+}^{3}}{ }^{i} \Sigma^{0}\left(x, \xi ; v_{j k}\right) E(x) g(x) d x \text { and } \\
\Sigma^{ \pm}\left(v_{j k}\right) g(p) & =\int_{\mathbf{R}_{+}^{3}}{ }^{i} \Sigma^{ \pm}\left(x, p ; v_{j k}\right) E(x) g(x) d x \\
& =Q( \pm j){ }^{i} W^{ \pm}\left(p ; v_{j k}\right) \int_{\mathbf{R}_{+}^{3}}{ }^{i} \Sigma\left(x, \xi ; v_{j k}\right) E(x) g(x) d x
\end{aligned}
$$

Some routine formulae are required for computations. We state these in the following lemma. The proof is an elementary application of complex function theory.

LEMMA 5.4. With $W^{ \pm}\left(p ; v_{j k}\right)$ of (5.39),

$$
\begin{gathered}
\int_{-\infty}^{\infty} \frac{d \eta}{\eta^{2}+\tau_{k}^{0^{2}}}=\frac{\pi}{\tau_{k}^{0}} \\
\int_{-\infty}^{\infty} W^{ \pm}\left(p ; v_{j k}\right)^{\bar{t}} W^{ \pm}\left(p ; v_{j k}\right) d \eta=4 a \pi\left[I-\frac{\sin \left(2 a \tau_{k}\right)}{2 a \tau_{k}} Q_{a}\right] \equiv 2 \pi \gamma_{k}(\xi) \\
\text { and } \int_{-\infty}^{\infty} \frac{{ }^{\bar{\tau}} W^{ \pm}\left(p ; v_{j k}\right)}{\eta+i \tau_{k}^{0}} d \eta=0 .
\end{gathered}
$$

The next lemma follows from (5.43) and (5.44).

LEMMA 5.5. If $g \in \mathscr{D}\left(\mathbf{R}_{-a}^{3}\right)$, then $\Sigma^{0}\left(v_{j k}\right) g(p) \in \mathscr{K}^{0}$ and $\Sigma^{ \pm}\left(v_{j k}\right) g(p) \in \mathscr{K}$.

The method of analysis we have used is based on writing the explicit Green's function in a form which represents the various "parts" (trapped modes, Alfven modes, etc.) of a solution. The idea is then to write the spectral resolution in terms of these parts. The following theorem gives this representation and, in addition, gives at least an abstract method for deciding how to (or how not to) "launch" a particular type of wave. Recalling (5.7), (5.44), (5.45), (5.30) and (5.31), we obtain the following.

THEOREM 5.6. Let $F(\cdot)$ denote the (right continuous) spectral measure for $\Lambda^{\prime}$ and let $g \in \mathscr{D}\left(\mathbf{R}_{-a}^{3}\right)$. Then for any finite interval $(a, b) \in \mathbf{R} \backslash\{0\}$,

$$
|F(a, b) g|^{2}=\sum_{j= \pm 1}\left\{\int_{\left(j c_{0}|p| \in(a, b)\right\}}\left|\Psi_{j 2}^{0} g(p)\right|_{E_{0}}^{2} d p+\int_{\left\langle j c_{0} p_{2} \in(a, b)\right\}}\left|\Psi_{j}^{0 \pm} g(p)\right|_{E_{0}}^{2} d p\right.
$$




$$
\begin{aligned}
& +\int_{\left.U j p_{2} \in(a, b)\right\}}\left|\Psi_{j}^{ \pm} g(p)\right|_{E}^{2} d p \\
& \left.+\sum_{k \in \mathbf{Z}_{+}} \int_{\left(j v_{k} \in(a, b)\right\}}\left(\left|\Sigma^{0}\left(v_{j k}\right) g(p)\right|_{E_{0}}^{2}+\left|\Sigma^{ \pm}\left(v_{j k}\right) g(p)\right|_{E}^{2}\right) d p\right\} .
\end{aligned}
$$

Launching a trapped mode (only), for example, depends on selecting an initial state in the subspace defined by the projector in the last term of (5.48). If no Alfven waves are wanted, we can avoid generating them by choosing data in the complement of the subspace generated by the projector in the second term of (5.48), etc.

The next result is obtained by polarization from (A1.12) and (5.48).

COROLLARY 5.7. Let $P_{0}$ denote the projection onto the null space of $\Lambda^{\prime}$ in $\mathscr{K}$ and set $\overline{\mathscr{K}}=\left(I-P_{0}\right) \mathscr{K}$. Then for any $f, g \in \overline{\mathscr{K}}$ and any interval $\Delta \subset \mathbf{R}$,

$$
\begin{aligned}
& (F(\Delta) f, g)=\sum_{j= \pm 1}\left\{\int_{\left(j c_{0}|p| \in \Delta\right\}}{ }^{i} \Psi_{j 2}^{0} f(p) E_{0} \Psi_{j 2}^{0} g(p) d p\right. \\
& +\int_{\left(j c_{0} p_{2} \in \Delta\right)}{ }^{i} \Psi_{j}^{0 \pm} f(p) E_{0} \Psi_{j}^{0 \pm} g(p) d p+\int_{\left(j c_{2} \in \Delta\right)}{ }^{\bar{t}} \Psi_{j}^{ \pm} f(p) E \Psi_{j}^{ \pm} g(p) d p \\
& +\sum_{k \in \mathbf{Z}_{+}} \int_{\left(j v_{k} \in \Delta\right]}\left({ }^{i} \Sigma^{0}\left(v_{j k}\right) f(p) E_{0} \Sigma^{0}\left(v_{j k}\right) g(p)\right. \\
& \left.\left.+{ }^{\overline{ }} \Sigma^{ \pm}\left(v_{j k}\right) f(p) E \Sigma^{ \pm}\left(v_{j k}\right) g(p)\right) d p\right\} \quad \text { and } \\
& (f, g)=\sum_{j= \pm 1}\left\{\left(\Psi_{j 2}^{0} f, \Psi_{j 2}^{0} g\right)_{\mathscr{E}^{0}}+\left(\Psi_{j}^{0} f, \Psi_{j}^{0} g\right)_{\mathscr{H}^{00}}+\left(\Psi_{j} f, \Psi_{j} g\right)_{\mathscr{H}}\right. \\
& \left.+\sum_{k \in \mathbf{Z}}\left[\left(\Sigma^{0}\left(v_{j k}\right) f, \Sigma^{0}\left(v_{j k}\right) g\right)_{\mathscr{H}^{0}}+\left(\Sigma^{ \pm}\left(v_{j k}\right) f, \Sigma^{ \pm}\left(v_{j k}\right) g\right)_{\mathscr{K}}\right]\right\} .
\end{aligned}
$$

In order to define eigenprojectors associated with $\Lambda^{\prime}$, we need the adjoints of the generalized transforms in (5.49).

PROPOSITION 5.8. Suppose $f \in L_{2}\left(\mathbf{R}^{3}, \mathbf{C}^{6}\right)$ has compact support. Then for $j= \pm 1$, $k \geq 0$, we define

$$
\begin{gathered}
\Psi_{j 2}^{0 *} f=\int_{\mathbf{R}^{3}} \Psi_{j 2}^{0 \pm}(x, p) E_{0} f(p) d p, \quad \Psi_{j}^{0^{*} f}=\int_{\mathbf{R}^{3}} \Psi_{j}^{0 \pm}(x, p) E_{0} f(p) d p \\
\Psi_{j}^{*} f=\int_{\mathbf{R}^{3}} \Psi_{j}^{ \pm}(x, p) E f(p) d p, \quad \Sigma^{0}\left(v_{j k}\right)^{*} f=\int_{\mathbf{R}^{3}} \Sigma^{0}\left(x, p, v_{j k}\right) E_{0} f(p) d p \\
\Sigma^{ \pm}\left(v_{j k}\right)^{*} f=\int_{\mathbf{R}_{+}^{3}}{ }^{i} \Sigma^{ \pm}\left(x, p ; v_{j k}\right) E f(p) d p
\end{gathered}
$$


If $f \in \mathscr{K}$ and $\left\{f_{N}\right\} \subset L_{2}\left(\mathbf{R}^{3}, \mathbf{C}^{6}\right)$ is a sequence of compactly supported functions converging to $f$ in $\mathscr{H}^{0}$ and $\mathscr{H}$, then, in the topology of $\mathscr{K}$,

$$
\begin{gathered}
\Psi_{j 2}^{0 *} f=\lim _{N \rightarrow \infty} \Psi_{j 2}^{0{ }^{*}} f_{N}, \quad \Psi_{j}^{0^{*}} f=\lim _{N \rightarrow \infty} \Psi_{j}^{0 \pm{ }^{*}} f_{N}, \\
\Psi_{j}{ }^{*} f=\lim _{N \rightarrow \infty} \Psi_{j}^{ \pm *} f_{N}, \quad \Sigma^{0}\left(v_{j k}\right)^{*} f=\lim _{N \rightarrow \infty} \Sigma^{0}\left(v_{j k}\right)^{*} f_{N}, \\
\Sigma^{ \pm}\left(v_{j k}\right)^{*} f=\lim _{N \rightarrow \infty} \Sigma^{ \pm}\left(v_{j k}\right)^{*} f_{N} .
\end{gathered}
$$

Furthermore, the ranges of $\Psi_{j 2}^{0 *}, \Psi_{j}^{0^{*}}, \Psi_{j}^{*}, \Sigma^{0}\left(v_{j k}\right)^{*}$ and $\Sigma^{ \pm}\left(v_{j k}\right)^{*}$ are contained in $\overline{\mathscr{K}}=\left(I-P_{0}\right) \mathscr{K}$.

LEMMA 5.9. For each $j= \pm 1, k \in \mathbf{Z}_{+}$, the operators

$$
\Pi\left(v_{j k}\right)=\Sigma^{0}\left(v_{j k}\right)^{*} \Sigma^{0}\left(v_{j k}\right)+\Sigma^{ \pm}\left(v_{j k}\right)^{*} \Sigma^{ \pm}\left(v_{j k}\right)
$$

are orthogonal orthoprojectors in $\overline{\mathscr{K}}$.

The assertion can be proved in an entirely analogous way to that found in [2, pp. 165-166]. We omit the details in the interest of space.

LemMa 5.10. Let $\bar{\Lambda}$ denote the part of $\Lambda^{\prime}$ in $\overline{\mathscr{K}}$. Then for $f \in D(\bar{\Lambda})$,

$$
\Psi_{j 2}^{0} \bar{\Lambda} f(p)=j c_{0}|p| \Psi_{j 2}^{0} f(p), \quad j= \pm 1 .
$$

LEMMA 5.11. Let $\bar{\Lambda}$ denote the part of $\Lambda^{\prime}$ in $\overline{\mathscr{K}}$. Then for $f \in D(\bar{\Lambda})$,

$$
\begin{array}{ll}
\Psi_{j}^{0} \bar{\Lambda} f(p)=j c_{0}|p| \Psi_{j}^{0} f(p), & j= \pm 1 \\
\Psi_{j} \bar{\Lambda} f(p)=j c_{0}|p| \Psi_{j} f(p), & j= \pm 1 .
\end{array}
$$

PROOF. The proof is similar to that of Lemma 4.9.

The projection operators in the resolution of the identity are computed in terms of the generalized eigenfunctions. This is the content of the following lemma.

LEMMA 5.12. Define the bounded operators in $\overline{\mathscr{K}}$ by

$$
\begin{aligned}
\Pi_{j 2} f & =\Psi_{j 2}^{0 *} \Psi_{j 2}^{0} f, \\
\Pi_{j}^{0} f & =\Psi_{j}^{0^{*}} \Psi_{j}^{0} f \quad \text { and } \\
\Pi_{j} f & =\Psi_{j}^{*} \Psi_{j} f .
\end{aligned}
$$

Then

$$
\Pi_{j^{\prime}\left(2 j^{\prime}\right)} \Pi\left(v_{j k}\right)=0, \quad j, j^{\prime}= \pm 1,
$$




$$
\begin{aligned}
\Pi_{j^{\prime}}^{(0)} \Pi_{j 2} & =0, \\
\Pi_{2} \Pi_{-2} & =0 \quad \text { and } \\
\Pi_{1}^{(0)} \Pi_{-1}^{(0)} & =0,
\end{aligned}
$$

where $\Pi_{j 2}$ and $\Pi_{j}$ are projections in $\overline{\mathscr{K}}, j= \pm 1$. Further, for any $f \in \overline{\mathscr{K}}$, there is the Parseval identity

$$
f=\sum_{j= \pm 1}\left[\Pi_{j} f+\Pi_{j}^{0} f+\Pi_{j 2} f+\sum_{k \in \mathbf{Z}_{+}} \Pi\left(v_{j k}\right) f\right],
$$

the components all being mutually orthogonal and the sum converging absolutely in the norm of $\overline{\mathscr{K}}$.

COROLLARY 5.13. For $f \in \overline{\mathscr{K}}$ the operators $\mathscr{P}_{r}, \mathscr{P}_{\sigma}$ defined by

$$
\mathscr{P}_{r} f=\left(\Pi_{2}+\Pi_{-2}+\Pi_{1}+\Pi_{-1}\right) f, \quad \mathscr{P}_{\sigma} f=\sum_{k \in \mathbf{Z}_{+}}\left[\Pi\left(v_{k}\right)+\Pi\left(v_{-k}\right)\right] f
$$

are mutually orthogonal orthoprojectors in $\overline{\mathscr{K}}$ such that

$$
\mathscr{P}=\mathscr{P}_{r}+\mathscr{P}_{\sigma},
$$

where $\mathscr{P}$ is the projection in $\mathscr{K}$ onto $\overline{\mathscr{K}}$. The space $\overline{\mathscr{K}}$ thus decomposes into the E-orthogonal direct sum

$$
\begin{aligned}
\overline{\mathscr{K}} & =\mathscr{P}_{r} \overline{\mathscr{K}} \oplus \mathscr{P}_{\sigma} \overline{\mathscr{K}} \\
& =\left[\Pi_{2} \overline{\mathscr{K}}+\Pi_{-2} \overline{\mathscr{K}}+\Pi_{1} \overline{\mathscr{K}}+\Pi_{-1} \overline{\mathscr{K}}\right] \bigoplus_{k \in \mathbf{Z}_{+}}\left[\Pi\left(v_{k}\right) \overline{\mathscr{K}}+\Pi\left(v_{-k}\right) \overline{\mathscr{K}}\right],
\end{aligned}
$$

all component subspaces being mutually E-orthogonal.

PROOF. (5.65) and (A1.7) show that for $f \in \overline{\mathscr{K}},\left|\mathscr{P}_{\sigma} f\right| \leq|f|$, and hence $\mathscr{P}_{\sigma}$ is continuous. It now follows that $\mathscr{P}_{\sigma}^{2}=\mathscr{P}_{\sigma}$ and $\mathscr{P}_{\sigma}^{*}=\mathscr{P}_{\sigma}$. The rest follows from the preceding results.

Finally, the main result concerns the make up of solutions to our problem. The previous two results with this theorem tell us among other things how to launch the various types of waves. This is given rigorously by the following theorem.

THEOREM 5.14. Solutions to (0.1) with the prescribed horizontal external field and the prescribed boundary condition, are delivered by a unitary group of operators. The solution is made up of orthogonal parts consisting of Alfven waves, trapped waves 
and "normal" waves. More formally, the orthogonal direct sum decomposition (5.64) reduces the part $\bar{U}(t)$ of the unitary group $U(t)=\exp \left(-i t \Lambda^{\prime}\right)$ in $\overline{\mathscr{K}}$ which has the representation

$$
\bar{U}(t) f(x)=\sum_{j= \pm 1}\left[\bar{U}(t) \Pi_{j 2} f(x)+\bar{U}(t) \Pi_{j} f(x)+\sum_{k \in \mathbf{Z}_{+}} \bar{U}(t) \Pi\left(v_{j k}\right) f(x)\right]
$$

with

$$
\bar{U}(t) \Pi_{j 2} f(x)=\Pi_{j 2} \bar{U}(t) f(x)=\Psi_{j 2}^{0} \exp \left(-i t j c_{0}|\cdot|\right) \Psi_{j 2}^{0} f(x)
$$

and similar formulae for the other $\Pi$. The waves $\left\{\bar{U}(t) \Pi_{j 2} f\right\},\left\{\bar{U}(t) \Pi_{j} f\right\}$ and $\left\{\bar{U}(t) \Pi\left(v_{j k}\right) f\right\}$ are pairwise orthogonal in $\overline{\mathscr{K}}$.

Further, $f \in D(\bar{\Lambda})$ if and only if $j c_{0}|p| \Psi_{j 2}^{0} f(p) \in \mathscr{H}^{0}, j c_{0} p_{2} \Psi_{j}^{0} f(p) \in$ $\mathscr{H}^{0}, j c p_{2} \Psi_{j} f(p) \in \mathscr{H}, j v_{k}(\xi) \Sigma^{0}\left(v_{j k}\right) f(p) \in L_{2}\left(\mathbf{R}^{3}, \mathbf{C}^{6}\right), j v_{k}(\xi) \Sigma^{ \pm}\left(v_{j k}\right) f(p) \in$ $L_{2}\left(\mathbf{R}^{3}, \mathbf{C}^{6}\right)$ and for such $f$ (using an abusive but convenient notation)

$$
\begin{aligned}
\bar{\Lambda} f(x)= & \sum_{j= \pm 1}\left[\Psi_{j 2}^{0 *} j c_{0}|p| \Psi_{j 2}^{0} f(x)+\Psi_{j}^{0^{*} j} c_{0} p_{2} \Psi_{j}^{0} f(x)+\Psi_{j}^{*} j c p_{2} \Psi_{j} f(x)\right. \\
& \left.+\sum_{k \in \mathbf{Z}_{+}}\left\{\Sigma^{0}\left(v_{j k}\right)^{*} j v_{k}(\xi) \Sigma^{0}\left(v_{j k}\right) f(x)+\Sigma^{ \pm}\left(v_{j k}\right)^{*} j v_{k}(\xi) \Sigma^{ \pm}\left(v_{j k}\right) f(x)\right\}\right],
\end{aligned}
$$

where the series converges absolutely in the sense of $\overline{\mathscr{K}}$. For $f \in D(\bar{\Lambda})$, the solution of the problem (2.1)-(2.3) is given by (5.68) ( $\bar{\Lambda}$ denotes the part of $\Lambda$ in $\overline{\mathscr{K}})$.

\section{Conclusion}

The structure of the solution (0.1) is rather simple, but considerable analysis is required to get to this conclusion. Considering the complexity of this work, it is likely that for the MHD system with non-constant pressure, much greater computational difficulties will arise. For instance, the propagation speeds involve nested roots and, therefore, the functions $\tau$ are more complicated, requiring more complex branch cuts. In addition, the expressions describing the reflection and transmission terms will be of considerably greater complexity. The present problem, while interesting in its own right, is extremely useful as a guide in working with the non-constant pressure case. It would also be interesting to study the case of internal layers in a fluid of the type studied here as well as for the case of a liquid semiconductor [7]. In the case of a liquid semiconductor, it is possible to formulate the problem of two half-spaces, one having nonzero conductivity. This is also an interesting challenge since the propagation 
speeds in the conducting half-space would be described by certain irreducible quintic and cubic polynomials and hence it is difficult or impossible to gain direct access to the propagation speeds themselves. We hope to examine these problems elsewhere. We conjecture at this point though that the solution of the layer problem for the full MHD system will display a structure very much like that seen here.

\section{Appendix I. Proofs}

Proofs, and in some cases, outlines of proofs for a number of the theorems follow.

Proof of Theorem 4.1. We show first that the roots of $J$ and $K$ are real. Suppose that $K\left(\xi, z_{1}\right)=0$ with $\operatorname{Im}\left(z_{1}\right) \neq 0$. Then since $\tau_{1}=c^{-1}\left(z_{1}^{2}-c^{2}|\xi|^{2}\right)^{1 / 2}$ and $\tau_{1}^{0}=$ $c_{0}^{-1}\left(z_{1}^{2}-c_{0}^{2}|\xi|^{2}\right)^{1 / 2}$ are real only on the branch cuts, we have $\operatorname{Im}\left(\tau_{1}\right)>0$ and $\operatorname{Im}\left(\tau_{1}^{0}\right)>0$. We may form the functions

$$
\begin{aligned}
f\left(x, \xi ; z_{1}\right)= & \left\{-x_{+}\left(x_{3}\right) e^{i \tau_{1}^{0} x_{3}} P^{06}\left(\xi, z_{1}, \tau_{1}^{0}\right) s\left(a \tau_{1}\right)\left(|\xi|^{2}+\tau_{1}^{0^{2}}\right) \tau_{1}\right. \\
& \left.+\chi_{-}\left(x_{3}\right) Q\left(\tau_{1}\left(x_{3}+a\right)\right)\left(|\xi|^{2}+\tau_{1}^{2}\right) P^{6}\left(\xi, z_{1},-\tau_{1}\right) \tau_{1}^{0}\right\} e^{i x^{\prime} \xi}
\end{aligned}
$$

and

$$
F\left(x ; z_{1}\right)=\int_{\mathbf{R}^{2}} f\left(x, \xi ; z_{1}\right) \phi(\xi) d \xi .
$$

Here $P^{6}$ indicates the 6th column of $P$. Then $F\left(0, z_{1}\right) \in L_{2}\left(\mathbf{R}_{-a}^{3}, \mathbf{C}^{6}\right), \mathfrak{B} F\left(x^{\prime},-a\right.$; $\left.z_{1}\right)=0$ and $L F\left(x^{\prime}, 0-; z_{1}\right)=L F\left(x^{\prime}, 0+; z_{1}\right)$ since $K\left(\xi, z_{1}\right)=0$. Further, $\left[\Lambda^{0}(D)-\right.$ $\left.z_{1}\right] F\left(x ; z_{1}\right)=0$ for $x_{3}>0$ and $\left[\Lambda(D)-z_{1}\right] F\left(x ; z_{1}\right)=0$ for $x_{3} \in(-a, 0)$. Set $G_{+}=A F(x), x_{3}>0, G_{-}=A F(x), x_{3} \in(-a, 0)$ and $G=\chi_{+} G_{+}+\chi_{-} G_{-}$; then $G \in L_{2}\left(\mathbf{R}_{-a}^{3}, \mathbf{C}^{6}\right)$ and, for $\psi \in \mathscr{D}\left(\mathbf{R}_{-a}^{3}, \mathbf{C}^{6}\right)$,

$$
\begin{aligned}
\langle F, A \psi\rangle= & \int_{\mathbf{R}_{+}^{3}}{ }^{i} F(x) A \psi(x) d x+\int_{\mathbf{R}^{2} \times(-a, 0)}{ }^{\bar{t}} F(x) A \psi(x) d x . \\
= & \langle G, \psi\rangle+i \int_{\mathbf{R}^{2}}\left\{A_{3}\left[F\left(x^{\prime}, 0+; z_{1}\right)-F\left(x^{\prime}, 0-; z_{1}\right)\right]\right\} \psi\left(x^{\prime}, 0\right) d x^{\prime} \\
& -\int_{\mathbf{R}^{2}}\left[A_{3} F\left(x^{\prime},-a ; z_{1}\right) \psi\left(x^{\prime},-a\right)\right] d x^{\prime} \\
= & \langle G, \psi\rangle .
\end{aligned}
$$

Therefore $A f=G \in L_{2}\left(\mathbf{R}_{-a}^{3}, \mathbf{C}^{6}\right)$ and so $F \in D\left(\Lambda^{\prime}\right)$. The same computation gives $\left[\Lambda^{\prime}-z_{1}\right] F=0$ which implies that $z_{1} \in \sigma\left(\Lambda^{\prime}\right)$; this is impossible sinse $\sigma\left(\Lambda^{\prime}\right) \subset \mathbf{R}$. 
If it is supposed that $J\left(\xi, z_{1}\right)=0$ and $\operatorname{Im}\left(z_{1}\right) \neq 0$, then the argument is the same but with

$$
\begin{aligned}
f\left(x, \xi ; z_{1}\right)= & \left\{\chi_{+}\left(x_{3}\right) e^{i \tau_{1}^{0} x_{3}} P^{03}\left(\xi, z_{1}, \tau_{1}^{0}\right) \tau_{1}^{2} c\left(a \tau_{1}\right)\left(p_{1}^{2}+\tau_{1}^{0^{2}}\right)\right. \\
& \left.-\chi_{-}\left(x_{3}\right) Q\left(\tau_{1}\left(x_{3}+a\right)\right)\left(p_{1}^{2}+\tau_{1}^{2}\right) \tau_{1}^{0^{2}} P^{3}\left(\xi, z_{1},-\tau_{1}\right)\right\} e^{i x^{\prime} \xi} .
\end{aligned}
$$

It is clear that $J$ and $K$ can vanish for $z \in \mathbf{R}$ only if $n>1$ and $\operatorname{Re}(z)=k \epsilon$ $\left(-c_{0}|\xi|,-c|\xi|\right) \cup\left(c|\xi|, c_{0}|\xi|\right)$, where $\tau^{0}(\xi, k)=\left.i c_{0}^{-1}\left|c_{0}^{2}\right| \xi\right|^{2}-\left.k^{2}\right|^{1 / 2}=i c_{0}^{-1}\left|\tau^{0}\right|$ and $\tau(\xi, k \pm i 0)= \pm\left.\left. c^{-1}\left|k^{2}-c^{2}\right| \xi\right|^{2}\right|^{1 / 2}= \pm|\tau|$. The case of $k$ in either interval is covered by considering the intersection of the branches of (note that $p_{1}^{2}-\left|\tau^{0}\right|^{2}=k^{2} c_{0}^{-2}-p_{2}^{2} \neq 0$ since $p \notin \beta$ )

$$
\frac{\left|\tau^{0}\right|}{p_{1}^{2}-\left|\tau^{0}\right|^{2}}=-\frac{\mu|\tau| \cot (a \tau)}{\mu_{0}\left(p_{1}^{2}+|\tau|^{2}\right)}
$$

for $K=0$, and

$$
\frac{\left|\tau^{0}\right|\left(|\xi|^{2}-\left|\tau^{0}\right|^{2}\right)}{p_{1}^{2}-\left|\tau^{0}\right|^{2}}=-\frac{\rho_{0}|\tau|\left(|\xi|^{2}+|\tau|^{2}\right) \cot (a \tau)}{\rho\left(p_{1}^{2}+|\tau|^{2}\right)}
$$

for $J=0$ in the first quadrant of $\mathbf{R}^{2}$ with the ellipse

$$
c_{0}^{2}\left|\tau^{0}\right|^{2}+c^{2}|\tau|^{2}=\left(c_{0}^{2}-c^{2}\right)|\xi|^{2} .
$$

The $j$ th roots $\pm v_{j}$ and $\pm w_{j}$ of $J$ and $K$ respectively, determined by the points of intersection $\left(\tau_{j v}^{0}, \tau_{j v}\right)$ and $\left(\tau_{j w}^{0}, \tau_{j w}\right)$ of (A1.3) with the $(j+1)$ st branch of (A1.2) and (A1.1) $(j=0,1, \ldots)$, are found by computing

$$
\left(v_{j}\right)^{2}=\frac{c_{0}^{2}}{n^{2}-1}\left[\left|\tau_{j v}^{0}\right|^{2}+\left|\tau_{j v}\right|^{2}\right] \text { and }\left(w_{j}\right)^{2}=\frac{c_{0}^{2}}{n^{2}-1}\left[\left|\tau_{j w}^{0}\right|^{2}+\left|\tau_{j w}\right|^{2}\right]
$$

at the points of intersection.

It is clear that $v_{j}$ and $w_{j}$ are increasing functions of $\xi_{1}$ and $\xi_{2}$ and (4.1) is geometrically obvious. Note that $c^{-2} v_{j}^{2}\left(w_{j}^{2}\right)=|\xi|^{2}\left(1+\left(\tau_{j v(j w)}^{2}\right) /|\xi|^{2}\right)$ and $\tau_{j v(j w)}$ is bounded as $|\xi| \rightarrow \infty$. This completes the proof.

Outline of the proof of Theorem 4.2. Let $g \in \mathscr{K}$; set

$$
\begin{aligned}
G(z) g(x) & =G_{+}(z) g(x)+G_{-}(z) g(x) \\
& =\int_{\mathbf{R}_{+}^{3}} G_{+}(x, y ; z) g(y) d y+\int_{\mathbf{R}^{2} \times(-a .0)} G_{-}(x, y ; z) g(y) d y .
\end{aligned}
$$


Writing out the expressions for $G_{ \pm}(z) g$ from $(4.6),(4.14),(4,29)-(4.33)$ and using Appendix II and noting that, for fixed $z$ and large $|\xi|, \tau(\xi, z)$ and $\tau^{0}(\xi, z)$ are equal to $i|\xi|+o(1)$, it is possible to estimate each term with the result that

$$
|G(z) g| \leq|\operatorname{Im}(z)|^{-2}|g|^{2}+C^{\prime}(z) \int_{-a}^{\infty}\left|\Phi_{2} g\left(\circ, y_{3}\right)\right|_{L_{2}}^{2} d y_{3}=C(z)|g|^{2},
$$

and hence $G(z)$ is a bounded operator on $\mathscr{K}$. We must now show that $G(z) g$ is in the domain of $\Lambda^{\prime}$. Choose functions $\phi$ and $\psi \in \mathscr{D}\left(\mathbf{R}_{-a}^{3}, \mathbf{C}^{6}\right)$, so that $\Phi_{2} \phi\left(0, y_{3}\right)$ is rapidly decreasing in $|\xi|$; then, setting $\phi_{ \pm}=\chi_{ \pm} \phi$,

$$
\begin{aligned}
\left\langle A \psi, G_{+}(z) \phi\right\rangle= & \left(\psi,\left[\phi_{+}+z G_{+}(z) \phi\right]\right)+i \int_{\mathbf{R}^{2}} d x^{\prime i} \psi\left(x^{\prime}, 0\right) \int_{\mathbf{R}_{-a}^{3}} A_{3}\left\{\left[S\left(x^{\prime}, 0, y ; z\right)\right.\right. \\
& \left.\left.-R^{0}\left(x^{\prime}, 0+, y ; z\right)+T\left(x^{\prime}, 0-, y ; z\right)\right] \phi(y)\right\} d y \\
= & \left(\psi,\left[\phi_{+}+z G_{+}(z) \phi\right]\right)
\end{aligned}
$$

by (4.13). Similarly, $\left\langle A \psi, G_{-}(z) \phi\right\rangle=\left(\psi,\left[\phi_{-}+z G_{-}(z) \phi\right]\right)$.

Therefore, $A G(z) f=E[\phi+z G(z) \phi] \in L_{2}\left(\mathbf{R}_{-a}^{3}, \mathbf{C}^{6}\right)$ and $\mathfrak{B} G(z) \phi\left(x^{\prime},-a\right)=0$ by (4.8) and (4.27). That is, $G(z) \phi \in D\left(\Lambda^{\prime}\right)$ and $\left[\Lambda^{\prime}-z I\right] G(z) \phi=\phi$. Applying $G(z)$ to both sides of this equation we have $G(z) \phi=G(z) \phi$. Thus $G(z)$ and $G(z)$ agree on a dense set, are both continuous, and therefore coincide everywhere. This completes the proof.

Proof of Lemma 5.2. First note that

$$
\begin{aligned}
J(\xi, z)= & \tau \cos (a \tau) \rho_{0}\left(p_{1}^{4}+p_{1}^{2} p_{2}^{2}+\tau^{2} p_{1}^{2}+\tau^{0^{2}} p_{1}^{2}+\tau^{0^{2}} p_{2}^{2}+\tau^{2} \tau^{0^{2}}\right) \\
& -i \rho \sin (a \tau) \tau^{0}\left(p_{1}^{4}+p_{1}^{2} \tau^{2}+p_{1}^{2} p_{2}^{2}+p_{2}^{2} \tau^{2}+\tau^{0^{2}} p_{1}^{2}+\tau^{0^{2}} \tau^{2}\right) \\
= & \tau \cos (a \tau) \rho_{0}\left(p_{1}^{2}+\tau^{0^{2}}\right)\left(p_{1}^{2}+p_{2}^{2}+\tau^{2}\right) \\
& -i \rho \sin (a \tau) \tau^{0}\left(p_{1}^{2}+\tau^{2}\right)\left(p_{1}^{2}+p_{2}^{2}+\tau^{0^{2}}\right) \\
= & \tau \cos (a \tau) \rho_{0}\left(p_{1}^{2}+\tau^{0^{2}}\right)\left(|\xi|^{2}+\tau^{2}\right) \\
& -i \rho \sin (a \tau) \tau^{0}\left(p_{1}^{2}+\tau^{2}\right)\left(|\xi|^{2}+\tau^{0^{2}}\right) \\
= & \frac{z^{2}}{\mu \mu^{0} c_{0}^{2} c^{2}}\left[\mu \tau \cos (a \tau)\left(p_{1}^{2}+\tau^{0^{2}}\right)-i \mu_{0} \tau^{0} \sin (a \tau)\left(p_{1}^{2}+\tau^{2}\right)\right] \\
= & \frac{z^{2}}{\mu \mu^{0} c_{0}^{2} c^{2}} K(\xi, z)
\end{aligned}
$$

the second to last equation resulting from the definitions of $\tau, \tau^{0}, c_{0}$ and $c$. 
We have

$$
\begin{aligned}
\partial_{2} K= & \frac{z \tau}{c_{0}^{2} c^{2}}\left(2 \mu \tau^{2}+c_{0}^{2} \mu\left(p_{1}^{2}+\tau^{0^{2}}\right)-2 i c_{0}^{2} a \mu_{0} \tau^{0}\left(p_{1}^{2}+\tau^{2}\right)\right) \cos (a \tau) \\
& +\frac{z}{c_{0}^{2} c^{2} \tau^{0}}\left(-a \mu c_{0}^{2}\left(p_{1}^{2}+\tau^{0^{2}}\right)-i c^{2} \mu_{0}\left(p_{1}^{2}+\tau^{2}\right)-i c_{0}^{2} \mu_{0} \tau^{0^{2}}\right) \sin (a \tau)
\end{aligned}
$$

at $z=v_{j k}, \partial_{z} K=0$ implies that $\left(p_{1}^{2}-\tau^{0^{2}}\right) \sin (a \tau)=0$, which is impossible, since $p \notin \beta$ and $K\left(\xi, v_{j k}\right)=0$. Hence, the zeroes of $K$ and $J$ are simple. This completes the proof.

Suggestion of the proof of Theorem 5.6. Note that the inner sum is actually finite because $(a, b)$ is bounded.

First, consider the first integral in (5.7)

$$
\begin{aligned}
I & =\pi^{-1} \int_{\mathbf{R}^{3}} \sum_{j= \pm 1} \int_{a}^{b} \frac{\varepsilon}{\left[\lambda_{j 2}^{0}(p)-\lambda\right]^{2}+\varepsilon^{2}}\left|\Psi_{j 2}^{0} f(p ; \lambda \pm i \varepsilon)\right|_{E_{0}}^{2} d \lambda d p \\
& =\pi^{-1} \int_{\mathbf{R}^{3} \cap\{|\eta|>\delta \mid} d p+\pi^{-1} \int_{\mathbf{R}^{3} \cap\{|\eta|<\delta\}} d p .
\end{aligned}
$$

We want to take the limit under the integral sign. The first integral is not difficult. The problem arises in the second integral where $|\eta|<\delta$ and $|\xi|$ is near $r_{k}$. In this region, the singularities of the integrand coalesce. For $|\eta|<\delta$ and $c_{0}|\xi|, c_{0}|p|, v_{k}(\xi) \in(a, b)$, a typical integral is now of the form

$$
\int_{a}^{b} \frac{\varepsilon \alpha(\xi, \lambda \pm i \varepsilon)}{\left|\tau^{0}\right|^{2}\left|\eta \pm \tau^{0}\right|^{2}\left|(\lambda \pm i \varepsilon)-v_{k}\right|^{2}} d \lambda
$$

where $\alpha(\xi, \lambda \pm i \varepsilon)$ is an element of $\left|z-v_{k}\right|^{2}{ }^{i} C^{0} C^{0}$ or $\left|z-v_{k}\right|^{2}{ }^{i} D^{0 \prime} D^{0 \prime}$. Suppose for example, that $\alpha(\xi, \lambda \pm i \varepsilon)=\alpha(\xi, z)=\left|z-v_{k}\right|^{2}\left|C_{66}^{0}\right|^{2}$ (see Appendix II) in a neighborhood of $\lambda=v_{k}(\xi)$.

Then

$$
\alpha(\xi, z)=\left|i \sin (a \tau) \mu_{0} \tau^{0}\left(p_{1}^{2}+\tau^{2}\right)+\mu \tau \cos (a \tau)\left(p_{1}^{2}+\tau^{0^{2}}\right)\right|^{2}|K|^{-2}\left|z-v_{k}\right|^{2} .
$$

For $z$ near $v_{k}$, we have

$$
\alpha(\xi, z)=\left|K^{\prime}\right|^{-2} 4 \mu_{0}^{2} \tau_{k}^{0^{2}}\left|p_{1}^{2}+\tau_{k}^{2}\right|^{2}\left|s\left(a \tau_{k}\right)\right|^{2}+o(1) .
$$

Further, it is easily seen (Theorem 4.1) that $\tau_{k}^{0} K^{\prime}$ is bounded away from zero for $|\xi|$ near $r_{k}$, so this together with (5.56) gives, for the critical portion of the interval $(a, b)$, a term proportional to

$$
\int_{v_{k}-\delta}^{v_{k}+\delta} d \lambda \frac{\varepsilon\left|\tau^{0}\right|^{2}}{\left|\eta \pm \tau^{0}\right|^{2}\left|z-v_{k}\right|^{2}}
$$


Since, with generic constant $\kappa$, for $\eta$ small both $\left|\tau^{0}\right|^{2} \leq \kappa\left|c_{0}^{-1} z-\right| \xi||$ and $\left|\eta \pm \tau^{0}\right|^{2} \geq$ $\kappa\left|c_{0}^{-1} z-\right| \xi||$, this integral is bounded by

$$
\int_{v_{k}-\delta}^{v_{k}+\delta} d \lambda \frac{\varepsilon}{\left(\lambda-v_{k}\right)^{2}+\varepsilon^{2}} \leq \pi
$$

Similarly, for other possible $\alpha$ it is found that the critical portion of the integral can always be reduced to one of the following forms:

$$
\int_{a}^{b} d \lambda \varepsilon /\left[\left|z-v_{k}\right|^{2}\right]_{z=\lambda \pm i \varepsilon} \leq \pi, \quad \int_{a}^{b} d \lambda \varepsilon /\left[\left|z-c_{0}\right| \xi||^{2}\right]_{z=\lambda \pm i \varepsilon} \leq \pi
$$

uniformly in $p$ in a neighborhood of $\eta=0$ and $\varepsilon \in\left(0, \varepsilon_{0}\right]$. It remains to evaluate the integral from the well-known formula

$$
\lim _{\varepsilon \rightarrow 0+} \pi^{-1} \int_{a}^{b} \frac{\varepsilon \phi(\lambda)}{(\lambda-v)^{2}+\varepsilon^{2}} d \lambda=\chi_{(a, b)}(\nu) \phi(\nu)
$$

$\left(\phi(\lambda)\right.$ is continuous). This results in the terms involving $\Sigma^{0}\left(v_{j k}\right) g(p), \Sigma^{ \pm}\left(v_{j k}\right) g(p)$, $\Psi_{j 2}^{0} g(p), \Psi_{j}^{0} g(p)$ and $\Psi_{j} g(p)$. We may obtain $\Sigma^{ \pm}\left(v_{j k}\right) g(p)$ from the second term in (5.7) recalling that $\Psi_{j 2}\left(x, p ; \lambda_{j 2}(p) \pm i 0\right)=0$. This completes the proof.

REMARK A1. If now $f \in \mathscr{K}$ is any compactly supported function and $\left\{f_{n}\right\} \subset$ $\mathscr{D}\left(\mathbf{R}_{-a}^{3}\right)$ converges to $f$ in $\mathscr{K}$, then (5.48) also holds for $f$. Let $P_{0}=F(0)-F(0-)$ be the projection onto the null space $N\left(\Lambda^{\prime}\right)$ of $\Lambda^{\prime}$ in $\mathscr{K}$. Then for any compactly supported $f \in \mathscr{K}$,

$$
\begin{aligned}
\left|\left(I-P_{0}\right) f\right|^{2}= & \sum_{j= \pm 1}\left\{\left\|\Psi_{j 2}^{0} f\right\|_{0}^{2}+\left\|\Psi_{j}^{0} f\right\|_{0}^{2}+\left\|\Psi_{j} f\right\|^{2}\right. \\
& \left.+\sum_{k \in \mathbf{Z}}\left[\left\|\Sigma^{0}\left(v_{k}\right) f\right\|_{0}^{2}+\left\|\Sigma^{ \pm}\left(v_{k}\right) f\right\|^{2}\right]\right\} .
\end{aligned}
$$

The sum now is infinite but converges since the left side is finite; the double bars denote the norms in $\mathscr{H}^{0}$ and $\mathscr{H}$. If $\chi_{M}$ is the characteristic function of the ball $\{x:|x| \leq M\}$, $M=1,2,3, \ldots$ and $f \in \mathscr{K}$, then, by (5.48), $\Psi_{j 2}^{0} \chi_{M} f, \Psi_{j}^{0} \chi_{M} f, \Psi_{j} \chi_{M} f, \Sigma^{0}\left(v_{k}\right) \chi_{M} f$ and $\Sigma^{ \pm}\left(v_{k}\right) \chi_{M} f$ are Cauchy sequences in $\mathscr{H}^{0}$ and $\mathscr{H}$ as appropriate. The maps

$$
\Psi_{j 2}^{0}, \quad \Psi_{j}^{0}, \quad \Psi_{j}, \quad \Sigma^{0}\left(v_{k}\right) \quad \text { and } \quad \Sigma^{ \pm}\left(v_{k}\right)
$$

defined by (5.35), (5.36), (5.30) and (5.31) on smooth compactly supported functions in $\mathscr{K}$ are bounded by one and thus extend by continuity to all of $\mathscr{K}$. The extended mappings we again denote by the same symbols. They are still bounded by one. 
Outline of proof of Proposition 5.8. Let $f \in L_{2}\left(\mathbf{R}^{3}, \mathbf{C}^{6}\right)$ have compact support which misses $\beta$ by a positive distance. Suppose also that $h \in \mathscr{D}\left(\mathbf{R}_{-a}^{3}\right)$. Then, for example,

$$
\begin{aligned}
\left(h, \Psi_{j 2}^{0 *} f\right) & =\left\langle\Psi_{j 2}^{0} h, f\right\rangle_{\mathscr{H}^{0}} \\
& =\int_{\mathbf{R}^{3}}\left\{\int_{\mathbf{R}_{-a}^{3}}{ }^{i} h(x) E(x) \Psi_{j 2}^{0 \pm}(x, p) d x\right\} E_{0} f(p) d p .
\end{aligned}
$$

The function ${ }^{\overline{ }} h(x) E(x) \Psi_{j 2}^{0 \pm}(x, p)$ is absolutely integrable with respect to $x$ with $L_{1}$ norm depending in a bounded way on $p \in \operatorname{supp} f$. The order of integration may thus be interchanged. This shows (A1.12) is correct. The others are handled similarly. The fact that the operators $\Psi_{j 2}^{0}, \Psi_{j}^{0}, \Psi_{j}, \Sigma^{0}\left(v_{k}\right)$ and $\Sigma^{ \pm}\left(v_{k}\right)$ are bounded gives the statement following (A1.12). The last statement follows from (5.48): if $f \in P_{0} \mathscr{K}$, then

$$
0=\sum_{j= \pm 1}\left\{\left\|\Psi_{j 2}^{0} f\right\|_{0}^{2}+\left\|\Psi_{j}^{0} f\right\|_{0}^{2}+\left\|\Psi_{j} f\right\|^{2}+\sum_{k \in \mathbf{Z}}\left[\left\|\Sigma^{0}\left(v_{k}\right) f\right\|_{0}^{2}+\left\|\Sigma^{ \pm}\left(v_{k}\right) f\right\|^{2}\right]\right\}
$$

and hence $\Psi_{j 2}^{0} f=\Psi_{j}^{0} f=\Psi_{j} f=\Sigma^{0}\left(v_{k}\right) f=\Sigma^{ \pm}\left(v_{k}\right) f=0$. Thus, for example, if $h \in \mathscr{H}^{0}$ and $f \in P_{0} \mathscr{K}$, then $\left(f, \Psi_{j 2}^{0 *} h\right)=\left\langle\Psi_{j 2}^{0} f, h\right\rangle_{\mathscr{K}^{0}}=0$ and so the range of $\Psi_{j 2}^{0 * *}$ is in $\left(I-P_{0}\right) \mathscr{K}$; similarly for the other maps. Notice that it follows from (5.48) that if $J: \mathscr{H} \rightarrow \mathscr{H}^{0}$ is the identification map $J f=f$, then the range of $\Sigma^{0}\left(v_{j k}\right)$ is orthogonal to the range of $J \Sigma^{ \pm}\left(v_{j k}\right)$. This completes the proof.

Proof of Lemma 5.10. The multiplication operator $M_{j}$ in $\mathscr{H}^{0}, M_{j} f(p)=j c_{0}|p|$ $\times f(p)$, with domain $D\left(M_{j}\right)=\left\{f \in \mathscr{H}^{0}:|p| f(p) \in \mathscr{H}^{0}\right\}$, is selfadjoint and hence closed. Let $b(x) \in \mathscr{D}\left(\mathbf{R}^{3}\right)$ be a radial function $b(x)=b(|x|)$, such that $b(x)=1$ for $|x|<R$ and $b(x)=0$ for $|x|>R+1$. Let $f \in D(\bar{\Lambda})$. Then the following integration by parts is justified:

$$
\begin{aligned}
\Psi_{j 2}^{0} b \bar{\Lambda} f(p)= & \int_{\mathbf{R}_{-a}^{3}}{ }^{i} \Psi_{j 2}^{0 \pm}(x, p) E(x) b(x) \bar{\Lambda}(D) f(x) d x \\
= & \int_{\mathbf{R}_{+}^{3}}{ }^{\bar{T}} \Psi_{j 2}^{0 \pm}(x, p) E(x) b(x) \bar{\Lambda}(D) f(x) d x \\
& +\int_{\mathbf{R}^{2} \times(-a, 0)}{ }^{i} \Psi_{j 2}^{0 \pm}(x, p) E(x) b(x) \bar{\Lambda}(D) f(x) d x \\
= & i \int_{\mathbf{R}^{2}}{ }^{i}\left[A_{3} \Psi_{j 2}^{0 \pm}\left(x^{\prime}, 0+, p\right)-A_{3} \Psi_{j 2}^{0 \pm}\left(x^{\prime}, 0-, p\right)\right] b\left(x^{\prime}, 0\right) f\left(x^{\prime}, 0\right) d x^{\prime} \\
& +i \int_{\mathbf{R}^{2}}{ }^{i} \Psi_{j 2}^{0 \pm}\left(x^{\prime},-a, p\right) A_{3} b\left(x^{\prime},-a\right) f\left(x^{\prime},-a\right) d x^{\prime}
\end{aligned}
$$




$$
\begin{aligned}
& +\int_{\mathbf{R}_{+}^{3}}{ }^{i}\left[\Lambda^{0} \Psi_{j 2}^{0 \pm}(x, p)\right] E_{0} b(x) f(x) d x \\
& +\int_{\mathbf{R}^{2} \times(-a, 0)}{ }^{\bar{T}}\left[\Lambda \Psi_{j 2}^{0 \pm}(x, p)\right] E_{0} b(x) f(x) d x \\
& +i \int_{\mathbf{R}_{-a}^{3}}^{\bar{i}} \Psi_{j 2}^{0 \pm}(x, p) A(x /|x|) b^{\prime}(x) \bar{\Lambda}(D) f(x) d x \\
& =j c_{0}|p| \int_{\mathbf{R}_{-a}^{3}}{ }^{i} \Psi_{j 2}^{0 \pm}(x, p) E(x) b(x) \bar{\Lambda}(D) f(x) d x \\
& +i \int_{\mathbf{R}_{-a}^{3}}{ }^{i} \Psi_{j 2}^{0 \pm}(x, p) A(x /|x|) b^{\prime}(x) \bar{\Lambda}(D) f(x) d x \\
& =j c_{0}|p| \Psi_{j 2}^{0} b f(p)+i \Psi_{j 2}^{0} g_{R}(p),
\end{aligned}
$$

where $g_{R}(x)=A(x /|x|) b^{\prime}(x) f(x)$. Since as $R \rightarrow \infty$ the functions $g_{R}(x) \rightarrow 0$, $b \bar{\Lambda} f \rightarrow \bar{\Lambda} f$ in $\mathscr{K}$ and $\Psi_{j 2}^{0}$ is bounded, it follows that $\Psi_{j 2}^{0} g_{R} \rightarrow 0, \Psi_{j 2}^{0} b \bar{\Lambda} f \rightarrow$ $\Psi_{j 2}^{0} \bar{\Lambda} f$ in $\mathscr{H}^{0}$ and hence, in the topology of $\mathscr{H}^{0}$,

$$
\Psi_{j 2}^{0} \bar{\Lambda} f(p)=\lim _{R \rightarrow \infty} j c_{0}|p| \Psi_{j 2}^{0} b f(p) .
$$

Now $\Psi_{j 2}^{0} b f \rightarrow \Psi_{j 2}^{0} f$ in $\mathscr{H}^{0}$ and this together with (5.50) and the fact that $M_{j}$ is closed implies that $\Psi_{j 2}^{0} f \in D\left(M_{j}\right)$ and

$$
\Psi_{j 2}^{0} \bar{\Lambda} f(p)=j c_{0}|p| \Psi_{j 2}^{0} f(p)
$$

for $f \in D(\bar{\Lambda})$. If now $f$ is any element of $D(\bar{\Lambda})$, let $\left\{f_{n}\right\} \subset D(\bar{\Lambda})$ as in Proposition 3.12 of [8] which converges to $f$ in graph norm. We then have

$$
\begin{gathered}
\left\|\Psi_{j 2}^{0} \Lambda_{s} f-\Psi_{j 2}^{0} \Lambda_{s} f_{n}\right\|_{\mathscr{H}^{0}}^{2}+\left\|\Psi_{j 2}^{0} f-\Psi_{j 2}^{0} f_{n}\right\|_{\mathscr{H}^{\circ}}^{2} \\
\leq\left\|\Lambda_{s} f-\Lambda_{s} f_{n}\right\|_{\mathscr{H}^{0}}^{2}+\left\|f-f_{n}\right\|_{\mathscr{H}^{0}}^{2} \rightarrow 0
\end{gathered}
$$

as $n \rightarrow \infty$ and so by (5.66) we have, in the sense of $\mathscr{H}^{0}$,

$$
\Psi_{j 2}^{0} \bar{\Lambda} f(p)=\lim _{n \rightarrow \infty} \Psi_{j 2}^{0} \bar{\Lambda} f_{n}(p)=\lim _{n \rightarrow \infty} j c_{0}|p| \Psi_{j 2}^{0} f_{n}(p)=j c_{0}|p| \Psi_{j 2}^{0} f(p),
$$

since the graph of $M_{j}$ is closed. The proof of the lemma is complete.

Proof of Lemma 5.12. We write $\overline{\mathscr{K}}$ for $\mathscr{K} \cap N\left(\Lambda^{\prime}\right)^{\perp}$. Let $f \in \overline{\mathscr{K}}$ satisfy (3.14)(3.15). From (5.67), (5.35), (5.36) and the fact that $\left|v_{j k}\right| \leq c_{0}|\xi|$, it follows that $\Pi\left(v_{j k}\right) f \in D(\bar{\Lambda})$. Also from (5.35) and (5.36),

$$
\Phi_{2} \bar{\Lambda} \Pi\left(v_{j k}\right) f\left(\xi, x_{3}\right)=j v_{j k}(\xi) \Phi_{2} \Pi\left(v_{j k}\right) f\left(\xi, x_{3}\right)
$$


hence by (5.43)

$$
\Psi_{j \prime 2}^{0} \bar{\Lambda} \Pi\left(v_{j k}\right) f(p)=j v_{j k}(\xi) \Psi_{j^{\prime} 2}^{0} \Pi\left(v_{j k}\right) f(p) .
$$

Now the right-hand sides of both the above equations vanish for $|\xi|<r_{k}$ and $v_{k}(\xi)<$ $c_{0}|\xi|$ for $|\xi|>r_{k}$; subtracting the two equations thus gives $\Psi_{j^{\prime} 2}^{0} \Pi\left(v_{j k}\right) f=0$. Since the set of such $f$ is dense in $\overline{\mathscr{K}}$ and this product of operators is continuous, this establishes half of (5.48). The other half is entirely similar. Equiations (5.57)-(5.59) are established in the same manner as well. By (A1.12) for $f, g \in \overline{\mathscr{K}}$,

$$
\begin{aligned}
(f, g)= & \sum_{j= \pm 1}\left\{\left(\Psi_{j 2}^{0} f, \Psi_{j 2}^{0} g\right)_{\mathscr{H}^{0}}+\left(\Psi_{j}^{0} f, \Psi_{j}^{0} g\right)_{\mathscr{H}^{0}}+\left(\Psi_{j} f, \Psi_{j} g\right)_{\mathscr{H}}\right. \\
& \left.+\sum_{k \in \mathbf{Z}}\left[\left(\Sigma^{0}\left(v_{j k}\right) f, \Sigma^{0}\left(v_{j k}\right) g\right)_{\mathscr{H}^{0}}+\left(\Sigma^{ \pm}\left(v_{j k}\right) f, \Sigma^{ \pm}\left(v_{j k}\right) g\right)_{\mathscr{K}^{0}}\right]\right\} .
\end{aligned}
$$

Taking $f=g$ and using (A1.14), (A1.15) and (5.55) and the fact that the $\Pi$ are selfadjoint, we see that

$$
\sum_{k \in \mathbf{Z}_{+}}\left|\Pi\left(v_{j k}\right) f\right|^{2}<\infty
$$

Hence from (5.62)-(5.63), the idempotent property of the $\Pi$ follows. This completes the proof.

Suggestion of the proof of Theorem 5.14. We use (5.60) to obtain the RadonNikodým derivative of $F$. This gives the result from the abstract function calculus for selfadjoint operators (see [1]) together with the lemmas above (especially Lemma 5.10):

$$
\begin{aligned}
& \left(f, F_{\lambda} g\right)-\left(f, F_{b} g\right)=\sum_{j= \pm 1}\left\{\int_{\left.V c_{0} \mid p \in \in(b, \lambda)\right\}}{ }^{i} \Psi_{j 2}^{0} f(p) E_{0} \Psi_{j 2}^{0} g(p) d p\right. \\
& +\int_{\left\langle c_{0} p_{2} \in(b, \lambda)\right\}}{ }^{\bar{\tau}} \Psi_{j}^{0 \pm} f(p) E_{0} \Psi_{j}^{0 \pm} g(p) d p \\
& +\int_{\left(j c_{0} p_{2} \in(b, \lambda)\right\}}{ }^{\bar{T}} \Psi_{j}^{ \pm} f(p) E \Psi_{j}^{ \pm} g(p) d p \\
& +\sum_{k \in \mathbf{Z}_{+}} \int_{\left(j v_{k} \in(b, \lambda)\right]}\left[{ }^{i} \Sigma^{0}\left(v_{j k}\right) f(p) E_{0} \Sigma^{0}\left(v_{j k}\right) g(p)\right. \\
& \left.\left.+{ }^{\bar{t}} \Sigma^{ \pm}\left(v_{j k}\right) f(p) E \Sigma^{ \pm}\left(v_{j k}\right) g(p)\right] d p\right\} .
\end{aligned}
$$


Note that the last sum is finite. The first term can be written as ( $m \vee 0$ means the max of $m$ and 0 )

$$
\begin{aligned}
& \sum_{j= \pm 1} \int_{\left(j c_{0}|p| \in(b, \lambda)\right\}}{ }^{i} \Psi_{j 2}^{0} f(p) E_{0} \Psi_{j 2}^{0} g(p) d p \\
& =\chi_{\mathbf{R}_{+}}(\lambda) \int_{c_{0}^{-1} \vee 0}^{c_{0}^{-1} \lambda} \int_{\mathbf{S}^{2}}{ }^{\bar{T}} \Psi_{2}^{0} f(|p| \omega) E_{0} \Psi_{2}^{0} g(|p| \omega)|p|^{2} d \omega d|p| \\
& \quad+\chi_{\mathbf{R}_{-}}(\lambda) \int_{-c_{0}^{-1} \lambda}^{-c_{0}^{-1} b} \int_{\mathbf{S}^{2}}{ }^{i} \Psi_{-2}^{0} f(|p| \omega) E_{0} \Psi_{-2}^{0} g(|p| \omega)|p|^{2} d \omega d|p|
\end{aligned}
$$

with a similar expression for the second terms. It is permissible to make the change of variable $|\xi| \rightarrow v_{k}(\xi)$ in the last terms of (5.51). Let $u_{k}(\xi)$ denote the $\mathbf{C}^{1}$ inverse of $v_{k}(\xi)$ where the latter is assumed to be extended to the left of $r_{k}$ by $c_{0}|\xi|$. Set $\omega^{\prime}=(\cos \phi, \sin \phi) \in \mathbf{S}^{\mathbf{1}}$. Then (note that $\Sigma^{0}\left(v_{j k}\right) g(p)$ and $\Sigma^{ \pm}\left(v_{j k}\right) g(p)$ depend on $p_{3}$ in a trivial way by (5.38)-(5.39) and we may integrate this out)

$$
\begin{gathered}
\sum_{j= \pm 1} \int_{\left(j v_{k} \in(b, \lambda)\right\}}{ }^{i} \Sigma^{0}\left(v_{j k}\right) f(p) E_{0} \Sigma^{0}\left(v_{j k}\right) g(p)+{ }^{i} \Sigma^{ \pm}\left(v_{j k}\right) f(p) E \Sigma^{ \pm}\left(v_{j k}\right) g(p) \\
=\chi_{\mathbf{R}_{+}}(\lambda) \int_{b \vee 0}^{u_{k}(\lambda)} \int_{\mathbf{S}^{\prime}}{ }^{\bar{t}} \Sigma^{0}\left(v_{k}\right) f\left(|\xi| \omega^{\prime}\right) E_{0} \Sigma^{0}\left(v_{k}\right) g\left(|\xi| \omega^{\prime}\right) \\
\quad+{ }^{i} \Sigma^{ \pm}\left(v_{k}\right) f\left(|\xi| \omega^{\prime}\right) E \Sigma^{ \pm}\left(v_{k}\right) g\left(|\xi| \omega^{\prime}\right) d \phi d|\xi| \\
+\chi_{\mathbf{R}_{-}}(\lambda) \int_{u_{k}(-\lambda)}^{u_{k}(-b)} \int_{\mathbf{S}^{1}}{ }^{i} \Sigma^{0}\left(v_{-k}\right) f\left(|\xi| \omega^{\prime}\right) E_{0} \Sigma^{0}\left(v_{-k}\right) g\left(|\xi| \omega^{\prime}\right) \\
+{ }^{i} \Sigma^{ \pm}\left(v_{-k}\right) f\left(|\xi| \omega^{\prime}\right) E \Sigma^{ \pm}\left(v_{-k}\right) g\left(|\xi| \omega^{\prime}\right) d \phi d|\xi| .
\end{gathered}
$$

By Fubini's theorem the absolutely continuous function $\left(f, F_{\lambda} g\right)-\left(f, F_{b} g\right)$ has the derivative $d\left(f, F_{\lambda} g\right) / d \lambda$. The Radon-Nikodým theorem allows the expression of functions of $\bar{\Lambda}$ in the usual way. The formulae (5.68)-(5.70) now follow.

\section{Appendix II. Singularities of matrices}

I. First, the matrices $C^{0}, D^{\prime}, D^{0 \prime \prime}$ and $C$ are given.

$$
\begin{gathered}
C^{0}(\xi, z)=\operatorname{diag}\left(d_{1}, A, d_{3}, d_{4}, d_{5}, d_{6}\right) \text { and } D^{\prime}(\xi ; z)=\operatorname{diag}\left(k_{1}, A, k_{3}, k_{4}, k_{5}, k_{6}\right) \\
d_{1}=\frac{\rho_{0} \tau \cos (a \tau)\left(p_{1}^{2}+\tau^{0^{2}}\right)\left(|\xi|^{2}+\tau^{2}\right)+i \rho \sin (a \tau) \tau^{0}\left(p_{1}^{2}+\tau^{2}\right)\left(|\xi|^{2}+\tau^{0^{2}}\right)}{J} \\
d_{4}=\frac{i \sin (a \tau) \mu_{0} \tau^{0}\left(p_{1}^{2}+\tau^{2}\right)+\mu \tau \cos (a \tau)\left(p_{1}^{2}+\tau^{0^{2}}\right)}{K}
\end{gathered}
$$




$$
\begin{gathered}
d_{3}=-d_{1}, \quad d_{5}=d_{4}, \quad d_{6}=-d_{4} ; \\
k_{1}=\frac{\tau^{0} \mu_{0}\left(p_{1}^{2}+\tau^{2}\right) \rho_{0}^{2}\left(|\xi|^{2}+\tau^{2}\right)}{\mu \rho J}, \quad k_{3}=\frac{\tau^{0}}{\tau} k_{1}, \\
k_{4}=\frac{\rho_{0}\left(|\xi|^{2}+\tau^{2}\right) \mu_{0}^{2}\left(p_{1}^{2}+\tau^{2}\right) \tau^{0}}{\mu \rho K\left(|\xi|^{2}+\tau^{0^{2}}\right)}, \quad k_{5}=k_{4}, \quad k_{6}=\frac{\tau^{0}}{\tau} k_{4} .
\end{gathered}
$$

II. The limits of the foregoing matrices as $z \rightarrow \lambda_{j 2}^{0}(p) \pm i 0$ are:

$$
\begin{aligned}
J\left(\xi, \lambda_{j 2}^{0}(p) \pm i 0\right)= & \pm j\left[\rho_{0}\left(\chi_{3}\right) \cos \left(a \chi_{3}\right)\left(p_{1}^{2}+|\eta|^{2}\right)\left(|\xi|^{2}+\chi_{3}^{2}\right)\right. \\
& \left. \pm j i \rho \sin \left(a \chi_{3}\right)|\eta|\left(p_{1}^{2}+\chi_{3}^{2}\right)\left(|\xi|^{2}+|\eta|^{2}\right)\right], \\
K\left(\xi, \lambda_{j 2}^{0}(p) \pm i 0\right)= & i|\eta| \mu_{0}\left(p_{1}^{2}+\chi_{3}^{2}\right) \sin \left(a \chi_{3}\right) \\
& \mp j \mu \chi_{3}\left(p_{1}^{2}+|\eta|^{2}\right) \cos \left(a \chi_{3}\right),
\end{aligned}
$$$$
C^{0}\left(\xi, \lambda_{j 2}^{0}(p) \pm i 0\right)=C^{0}(p)=\operatorname{diag}\left(d_{1}, A, d_{3}, d_{4}, d_{5}, d_{6}\right) \text {, where }
$$

$$
\begin{aligned}
d_{1}= & \mp j\left(\rho_{0}\left(\chi_{3}\right) \cos \left(a \chi_{3}\right)\left(p_{1}^{2}+|\eta|^{2}\right)\left(|\xi|^{2}+\chi_{3}^{2}\right)\right. \\
& \left. \pm j i \rho \sin \left(a \chi_{3}\right)|\eta|\left(p_{1}^{2}+\chi_{3}^{2}\right)\left(|\xi|^{2}+|\eta|^{2}\right)\right) / J,
\end{aligned}
$$$$
d_{4}=-\left(i \mu_{0}(-j|\eta|)\left(p_{1}^{2}+\chi_{3}^{2}\right) \sin \left(a \chi_{3}\right) \mp j \mu_{\chi_{3}}\left(p_{1}^{2}+|\eta|^{2}\right) \cos \left(a \chi_{3}\right)\right) / K \text {, }
$$$$
d_{3}=-d_{1}, \quad d_{5}=-d_{4}, \quad d_{6}=d_{4} .
$$

Also $D^{\prime}\left(\xi, \lambda_{j 2}^{0}(p) \pm i 0\right)=\operatorname{diag}\left(k_{1}, A, k_{3}, k_{4}, k_{5}, k_{6}\right)$, where

$$
\begin{array}{ll}
k_{1}=\frac{( \pm j|\eta|) \mu_{0}\left(p_{1}^{2}+\chi_{3}^{2}\right) \rho_{0}^{2}\left(|\xi|^{2}+\chi_{3}^{2}\right)}{\mu \rho J}, & k_{3}=\frac{|\eta|}{ \pm j \tau} k_{1}, \\
k_{4}=\frac{\rho_{0}\left(|\xi|^{2}+\chi_{3}^{2}\right) \mu_{0}^{2}\left(p_{1}^{2}+\chi_{3}^{2}\right)( \pm j|\eta|)}{\mu \rho K\left(|\xi|^{2}+|\eta|^{2}\right)}, & k_{5}=k_{4}, \quad k_{6}=\frac{|\eta|}{ \pm j\left(\mp j \chi_{3}\right)} k_{4} .
\end{array}
$$

The matrix $D^{0}(p)$ of the text is now given by $Q^{\prime} D^{0 \prime}\left(\xi, \lambda_{j 2}^{0}(p) \pm i 0\right)$ (compare $D^{\prime}\left(\xi, \lambda_{j 2}^{0}(p) \pm i 0\right)$ ) and $D(p)$ is given by $Q^{\prime} D^{\prime}\left(\xi, \lambda_{j 2}^{0}(p) \pm i 0\right)$, with $Q^{\prime}=Q( \pm j)=$ $\operatorname{diag}( \pm j, A, 1, \pm j, \pm j, 1)$ if $n>1$ or $I$ if $n<1$. The matrix $Q^{0}(\phi)$ of the text is given by $\operatorname{diag}(s(\phi), A, c(\phi), s(\phi), s(\phi), c(\phi))$.

III. The matrices $D^{0 \prime \prime}(\xi, y, z)=\operatorname{diag}\left(h_{1}, A, h_{3}, h_{4}, h_{5}, h_{6}\right)$ and $C(\xi, y, z)=$ $\operatorname{diag}\left(g_{1}, A, g_{3}, g_{4}, g_{5}, g_{6}\right)$;

$$
\begin{aligned}
& g_{1}=-\frac{2 \mu \rho^{2} \tau\left(|\xi|^{2}+\tau^{0^{2}}\right)\left(p_{1}^{2}+\tau^{0^{2}}\right) \sin \left(\tau\left(a+y_{3}\right)\right) e^{i a \tau}}{\mu_{0} \rho_{0} J}, \\
& g_{3}=-\frac{2 \mu \rho^{2} \tau^{2}\left(|\xi|^{2}+\tau^{0^{2}}\right)\left(p_{1}^{2}+\tau^{0^{2}}\right) \cos \left(\tau\left(a+y_{3}\right)\right) e^{-i a \tau}}{\mu_{0} \rho_{0} \tau^{0} J},
\end{aligned}
$$




$$
\begin{gathered}
g_{4}=\frac{2 \mu^{2} \rho \tau\left(|\xi|^{2}+\tau^{0^{2}}\right) \sin \left(\tau\left(a+y_{3}\right)\right)\left(p_{1}^{2}+\tau^{0^{2}}\right) e^{i a \tau}}{\mu_{0} \rho_{0}\left(|\xi|^{2}+\tau^{2}\right) K}, \\
g_{5}=-\frac{2 i \mu^{2} \rho \tau\left(|\xi|^{2}+\tau^{0^{2}}\right) \sin \left(\tau\left(a+y_{3}\right)\right)\left(p_{1}^{2}+\tau^{2}\right)\left(-e^{-i a \tau}\right)}{\mu_{0} \rho_{0}\left(|\xi|^{2}+\tau^{2}\right) K}, \\
g_{6}=\frac{2 \mu^{2} \rho \tau^{2}\left(|\xi|^{2}+\tau^{0^{2}}\right) \cos \left(\tau\left(a+y_{3}\right)\right)\left(p_{1}^{2}+\tau^{0^{2}}\right)\left(-e^{-i a \tau}\right)}{\mu_{0} \rho_{0} \tau^{0}\left(|\xi|^{2}+\tau^{2}\right) K}, \\
h_{1}=i \sin \left(\tau\left(a+y_{3}\right)\right) F / J, \quad h_{3}=\cos \left(\tau\left(a+y_{3}\right)\right) F / J, \quad \text { where } \\
h_{4}=i \sin \left(\tau\left(a+y_{3}\right)\right) G / K, \quad h_{5}=h_{4}, \quad h_{6}=\cos \left(\tau\left(a+y_{3}^{2}\right)\right) G / K, \\
G=\mu_{0} \tau^{0}\left(p_{1}^{2}+\tau^{2}\right)-\mu \tau\left(p_{1}^{2}+\tau^{0^{2}}\right) .
\end{gathered}
$$

IV. The matrices $D^{0 \prime}(\xi, z)$ and $C^{\prime}(\xi, z)$ of (4.35)-(4.36) are now easily written down. They are diagonal matrices of the form $\operatorname{diag}\left(h_{1}^{\prime}, A, h_{3}^{\prime}, h_{4}^{\prime}, h_{5}^{\prime}, h_{6}^{\prime}\right)$ and $\operatorname{diag}\left(g_{1}^{\prime}, A, g_{3}^{\prime}\right.$, $\left.g_{4}^{\prime}, g_{5}^{\prime}, g_{6}^{\prime}\right)$, respectively;

$$
\begin{gathered}
h_{1}^{\prime}=-e^{i a \tau} \rho_{0} \tau_{0} F /(2 J \rho \tau), \quad h_{3}^{\prime}=-h_{1}^{\prime}, \quad \text { where } \\
F=-\tau^{0} \rho\left(p_{1}^{2}+\tau^{2}\right)\left(p_{1}^{2}+p_{2}^{2}+\tau^{0^{2}}\right)+\rho_{0} \tau\left(p_{1}^{2}+\tau^{0^{2}}\right)\left(p_{1}^{2}+p_{2}^{2}+\tau^{2}\right), \\
h_{4}^{\prime}=-e^{i a \tau} \mu_{0} \tau^{0} G /(2 K \mu \tau), \quad h_{5}^{\prime}=h_{4}^{\prime}, \quad h_{6}^{\prime}=-h_{4}^{\prime}, \quad \text { where } \\
G=\mu_{0} \tau^{0}\left(p_{1}^{2}+\tau^{2}\right)-\mu \tau\left(p_{1}^{2}+\tau^{0^{2}}\right) .
\end{gathered}
$$

Also

$$
\begin{aligned}
& g_{1}^{\prime}=-i \frac{\mu \rho^{2} \tau\left(|\xi|^{2}+\tau^{0^{2}}\right)\left(p_{1}^{2}+\tau^{0^{2}}\right) e^{i a \tau}}{\mu_{0} \rho_{0} J}, \\
& g_{3}^{\prime}=-\frac{\mu \rho^{2} \tau^{2}\left(|\xi|^{2}+\tau^{0^{2}}\right)\left(p_{1}^{2}+\tau^{0^{2}}\right)\left(-e^{-i a \tau}\right)}{\mu_{0} \rho_{0} \tau^{0} J} \\
& g_{4}^{\prime}=-\frac{\mu^{2} \rho \tau\left(|\xi|^{2}+\tau^{0^{2}}\right)\left(p_{1}^{2}+\tau^{0^{2}}\right) e^{i a \tau}}{\mu_{0} \rho_{0}\left(|\xi|^{2}+\tau^{2}\right) K} \\
& g_{5}^{\prime}=\frac{\mu^{2} \rho \tau\left(|\xi|^{2}+\tau^{0^{2}}\right)\left(p_{1}^{2}+\tau^{2}\right)\left(-e^{-i a \tau}\right)}{\mu_{0} \rho_{0}\left(|\xi|^{2}+\tau^{2}\right) K} \\
& g_{6}^{\prime}=\frac{\mu^{2} \rho \tau^{2}\left(|\xi|^{2}+\tau^{0^{2}}\right)\left(p_{1}^{2}+\tau^{0^{2}}\right)\left(-e^{-i a \tau}\right)}{\mu_{0} \rho_{0} \tau^{0}\left(|\xi|^{2}+\tau^{2}\right) K}
\end{aligned}
$$

Referring to (2.15) we see that the required identities (4.34) are satisfied. 
V. The limits of the matrices as $z \rightarrow v_{j k}$ at $v_{j k} \pm i 0$ are:

$$
\begin{array}{ll}
C^{0}\left(\xi, v_{j k}\right)=\lim \left(z-v_{j k}\right) C^{0}(\xi, z), & D^{ \pm \ni}\left(\xi, v_{j k}\right)=\lim \left(z-v_{j k}\right) D^{\prime}(\xi, z), \\
D^{0}\left(\xi, v_{j k}\right)=\lim \left(z-v_{j k}\right) D^{0 \prime}(\xi, z), & C^{ \pm \prime}\left(\xi, v_{j k}\right)=\lim \left(z-v_{j k}\right) C^{\prime}(\xi, z) .
\end{array}
$$

In the paper, the matrices $D^{ \pm}\left(\xi, v_{j k}\right)$ and $C^{ \pm}\left(\xi, v_{j k}\right)$ are defined as

$$
\begin{aligned}
& D^{ \pm}\left(\xi, v_{j k}\right)=\left(i \tau_{j k}^{0}\right)^{-1} E_{0}^{-1} D^{ \pm \ni}\left(\xi, v_{j k}\right) E \text { and } \\
& C^{ \pm}\left(\xi, v_{j k}\right)=-Q\left({ }^{\circ} \pm j\right) e^{i a \tau_{j k}} \tau_{j k}^{-1} C^{ \pm \prime}\left(\xi, v_{j k}\right)
\end{aligned}
$$

Here $J^{\prime}$ and $K^{\prime}$ represent $\partial_{z} J$ and $\partial_{z} K$ (evaluated at $z=v_{j k} \pm i 0$ ). We have used the abusive but convenient notation of writing $\tau_{j k}^{0}$ for $\left|\tau_{j k}^{0}\right|$ and $\tau_{j k}$ for $\pm j \tau_{j k}$.

We have

$$
\begin{aligned}
& d_{1}=-2 \rho \sin \left(a \tau_{j k}\right) \tau_{j k}^{0}\left(p_{1}^{2}+\tau_{j k}^{2}\right)\left(|\xi|^{2}-\tau_{j k}^{0}\right) / J^{\prime}, \quad d_{3}=-d_{1}, \\
& d_{4}=-2 \sin \left(a \tau_{j k}\right) \mu_{0} \tau_{j k}^{0}\left(p_{1}^{2}+\tau_{j k}^{2}\right) / K^{\prime}, \quad d_{5}=d_{4}, \quad d_{6}=-d_{4}, \\
& k_{1}=\frac{i \tau_{j k}^{0} \mu_{0} \rho_{0}^{2}\left(p_{1}^{2}+\tau_{j k}^{2}\right)\left(|\xi|^{2}+\tau_{j k}^{2}\right)}{\mu \rho J^{\prime}}, \quad k_{3}=-i \frac{\tau_{j k}^{0}}{\tau_{j k}} k_{1}, \\
& k_{4}=\frac{i \rho_{0} \mu_{0}^{2} \tau_{j k}^{0}\left(|\xi|^{2}+\tau_{j k}^{2}\right)\left(p_{1}^{2}+\tau_{j k}^{2}\right)}{\mu \rho K^{\prime}\left(|\xi|^{2}-\tau_{j k}^{2^{2}}\right)}, \quad k_{5}=-i k_{4}, \quad k_{6}=\frac{\tau_{j k}^{0}}{\tau_{j k}} k_{5}, \\
& h_{1}^{\prime}=-i e^{i a \tau_{j k}} \rho_{0} \tau_{j k}^{0} F /\left(2 J^{\prime} \rho \tau_{j k}\right), \quad h_{3}^{\prime}=-h_{1}^{\prime} \text {, where } \\
& F=-i \tau_{j k}^{0} \rho\left(p_{1}^{2}+\tau_{j k}^{2}\right)\left(|\xi|^{2}-\tau_{j k}^{0}\right)+\rho_{0} \tau_{j k}\left(p_{1}^{2}-\tau_{j k}^{0}\right)\left(|\xi|^{2}+\tau_{j k}^{2}\right) \text {, } \\
& h_{4}^{\prime}=\frac{i e^{i a \tau_{j k}} \mu_{0} \tau_{j k}^{0}\left(i \mu_{0} \tau_{j k}^{0}\left(p_{1}^{2}-\tau_{j k}^{2}\right)-\mu \tau_{j k}\left(p_{1}^{2}-\tau_{j k}^{0}\right)\right)}{-2 K^{\prime} \mu \tau_{j k}}, \\
& h_{5}^{\prime}=\frac{i e^{i a \tau_{j k}} \mu_{0} \tau_{j k}^{0}\left(i \mu_{0} \tau_{j k}^{0}\left(p_{1}^{2}+\tau_{j k}^{2}\right)-\mu \tau_{j k}\left(p_{1}^{2}-\tau_{j k}^{0}\right)\right)}{-2 K^{\prime} \mu \tau_{j k}}, \quad h_{6}^{\prime}=-h_{5}^{\prime} \text {, } \\
& g_{1}^{\prime}=-i \frac{\mu \rho^{2} \tau_{j k}\left(|\xi|^{2}-\tau_{j k}^{0^{2}}\right)\left(p_{1}^{2}-\tau_{j k}^{0}\right) e^{i a \tau_{j k}}}{\mu_{0} \rho_{0} J^{\prime}}, \quad g_{3}^{\prime}=-i \frac{\tau_{j k}}{\tau_{j k}^{0}} g_{1}^{\prime}, \\
& g_{4}^{\prime}=-\frac{\mu^{2} \rho \tau_{j k}\left(|\xi|^{2}-\tau_{j k}^{0}\right)\left(p_{1}^{2}-\tau_{j k}^{0}\right) e^{i a \tau_{j k}}}{\mu_{0} \rho_{0}\left(|\xi|^{2}+\tau_{j k}^{2}\right) K^{\prime}}, \quad g_{5}^{\prime}=-i g_{4}^{\prime}, \quad g_{6}^{\prime}=-\frac{\tau_{j k}}{\tau_{j k}^{0}} g_{4}^{\prime} \text {. }
\end{aligned}
$$

The identities $|\xi|^{2}+\tau_{j k}^{2}=c^{-2} v_{j k}^{2}$ and $|\xi|^{2}-\tau_{j k}^{2}=c_{0}^{-2} v_{j k}^{2}$ are useful in simplifying computations with these matrices. 


\section{Acknowledgement}

This work was partially supported by a faculty research grant from Brigham Young University and the Centre National de Recherche Scientifique, France.

\section{References}

[1] N. Dunford and J. T. Schwartz, Linear Operators. Part II (Interscience, New York, 1963).

[2] D.'S. Gilliam and J. R. Schulenberger, "Electromagnetic waves in a three-dimensional half-space with a dissipative boundary", J. Math. Anal. Appl. 89 (1982) 129-185.

[3] T. Ikebe, "Eigenfunction expansions associated with the Schrödinger operator and their application to scattering theory", Arch. Rational Mech. Anal. 5 (1960) 1-34.

[4] P. Lax and R. S. Phillips, "Local boundary conditions for dissipative symmetric linear differential operators", Comm. Pure Appl. Math. 13 (1960) 427-455.

[5] A. E. Lifschitz, Magnetohydrodynamics and Spectral Theory (Kluwer, Dordrecht, 1989).

[6] W. V. Smith, "Waves in a perfectly conducting fluid filling a half-space", IMA J. Appl. Math. 43 (1989) 47-69.

[7] W. V. Smith, "The analysis of a model for wave motion in a liquid semiconductor: boundary interaction and variable conductivity", SIAM J. Math. Anal. 22 (1991) 352-378.

[8] W. V. Smith, "Wave motion in a conducting fluid with a boundary layer, I. Hilbert space formulation", J. Math. Anal. Appl. 188 (1994) 680-699.

[9] C. H. Wilcox, "Transient wave propagation in homogeneous anisotropic media", Arch. Rational Mech. Anal. 37 (1970) 323-343.

[10] C. H. Wilcox, "Asymptotic wave functions and energy distributions in strongly propagative anisotropic media”, J. Math. Pures et Appl. 57 (1978) 275-321. 